\title{
Observations of General Relativity at strong and weak limits
}

\author{
Gene G. Byrd, Arthur Chernin, Pekka Teerikorpi and Mauri Valtonen
}

\begin{abstract}
Einstein's General Relativity theory has been tested in many ways during the last hundred years as reviewed in this chapter. Two tests are discussed in detail in this article: the concept of a zero gravity surface, the roots of which go back to Järnefelt, Einstein and Straus, and the no-hair theorem of black holes, first proposed by Israel, Carter and Hawking. The former tests the necessity of the cosmological constant $\Lambda$, the latter the concept of a spinning black hole. The zero gravity surface is manifested most prominently in the motions of dwarf galaxies around the Local Group of galaxies. The no-hair theorem is testable for the first time in the binary black hole system OJ287. These represent stringent tests at the limit of weak and strong gravitational fields, respectively. In this article we discuss the current observational situation and future possibilities.
\end{abstract}

Keywords. General Relativity, relativity observational tests, dark energy, Local Group, KahnWoltjer, Coma Cluster of galaxies, black holes, quasars, OJ287.

AMS classification. 83, 85.

\section{Introduction}

In his theory of General Relativity, Einstein (1916) concluded that matter causes curvature in the surrounding spacetime, and bodies react to this curvature in such a way that there appears to be a gravitational attraction which causes acceleration. From the geometry of spacetime, it is possible to calculate the orbits of bodies which are influenced by gravity. In flat spacetime the force-free motion happens on a straight line, but in a spacetime curved by mass/energy the force free motion can create practically closed orbits as seen in the elliptical motion of a planet around the Sun.

The Einstein equations with the cosmological constant $\Lambda$ (Einstein 1917) have the form:

$$
R_{\mu \nu}-\frac{1}{2} R g_{\mu \nu}+\Lambda g_{\mu \nu}=-\frac{8 \pi G}{c^{4}} T_{\mu \nu}
$$

The Ricci tensor $R_{\mu \nu}$ and the Ricci scalar $R$ are functions of the metric tensor $g_{\mu \nu}$. The metric tensor describes the geometry of the spacetime while the Ricci tensor and the Ricci scalar measure its curvature. The energy momentum tensor $T_{\mu \nu}$ has the dominant

This work has been supported by The Finnish Society of Sciences and Letters and The Finnish Academy of Science and Letters. 
component $T_{00}=\rho_{M} c^{2}$ where $\rho_{M}$ is the matter density, $G$ is the gravitational constant and $c$ is the speed of light. See e.g. Byrd et al. $(2007,2012)$ for details.

In the presence of the cosmological constant $\Lambda$ the metric of a spherically symmetric object of mass $M$ is (Lemaitre 1931, McVittie 1932, 1933)

$$
\begin{array}{r}
d s^{2}=-\left(1-\frac{2 G M}{r c^{2}}-\frac{\Lambda r^{2}}{3}\right) d t^{2}+\left(1-\frac{2 G M}{r c^{2}}-\frac{\Lambda r^{2}}{3}\right)^{-1} d r^{2} \\
+r^{2}\left(d \theta^{2}+\sin ^{2} \theta d \phi^{2}\right)
\end{array}
$$

where $d s$ is the line element of the 4-dimensional spacetime, and $t, r, \theta, \phi$ are the spherical polar coordinates, centered on the body.

The deviation from the Minkowski "flat" metric is minimal when

$$
r=R_{V}=\left(\frac{3 G M}{\Lambda c^{2}}\right)^{1 / 3} .
$$

The surface defined by this radial distance from the center is called the zero gravity surface, and it is the weak field limit of General Relativity in the presence of the cosmological constant. It becomes significant in the smallest scales of cosmological expansion, such as in the Hubble flow around the Local Group of galaxies. Järnefelt (1933) was the first to derive Eq. 1.4 and the zero gravity surface is now understood to appear within the "vacuole" introduced by Einstein and Straus $(1945,1946)$ to describe the environments of bound mass concentrations in expanding space. This weak field limit is our first topic of testing General Relativity which we describe in detail.

The weak field is often also defined by (Psaltis 2008)

$$
v / c \ll 1, \quad r_{s} / r \ll 1
$$

while in case of a strong field we have

$$
v / c \sim 1, \quad r_{s} / r \sim 1 .
$$

Here $v$ is the orbital speed or other characteristic velocity, $r$ is the orbital radius or other characteristic length scale in the system and $r_{s}$ is the Schwarzschild radius of the primary body of mass $M$

$$
r_{s}=\frac{2 G M}{c^{2}} .
$$

In the first weak field category belong e.g. the precession of Mercury's orbit, bending of light near the Sun, precession of a binary pulsar orbit, gravitational radiation from the binary pulsar, relativistic geodetic precession and the precession due to the relativistic Lense-Thirring effect. In the latter strong field category we have at present 
only the binary black hole system OJ287, and hopefully in future other merging black hole binaries. The strong field limit in OJ287 is our second detailed topic of testing General Relativity.

Before these detailed discussions, we give a historical review of tests of General Relativity. Historically, the first test case supporting General Relativity was the nonNewtonian precession of the major axis of the planet Mercury. In Newtonian theory, a small object orbiting a concentrated spherically symmetric central body should retrace the same elliptical path repeatedly. In case of Mercury, the major axis of its elliptical orbit precesses slowly. The precession is mostly due to gravitation of other planets. However, the mathematician Urbain Le Verrier of Paris Observatory concluded, after the influence of other planets have been deducted, that there was a remaining observed shift of $38^{\prime \prime} /$ century in the perihelion of Mercury (Le Verrier 1859). He ascribed this to an unknown planet inside Mercury's orbit. This presumed planet was never found despite extensive searches. The United States Naval Observatory's Simon Newcomb (1895) recalculated the observed perihelion shift and obtained $41^{\prime \prime} \pm 2^{\prime \prime}$ /century (also see Hall 1894, Shapiro et al. 1976).

With General Relativity Einstein (1915) derived an additional precession term to be added to the Newtonian precession. Einstein's calculation gave a "post-Newtonian" (PN) force law which is almost but not quite the inverse square law proposed by Newton. For the post-Newtonian case, in one orbit, the ellipses traced by the two bodies orbiting each other precess through an angle given by

$$
\Delta \phi=\frac{6 \pi G M}{c^{2} a\left(1-e^{2}\right)}=3 \pi \frac{r_{s}}{a\left(1-e^{2}\right)},
$$

where $M$ is the total mass of the two bodies, $a$ is the semi-major axis of the relative motion and $e$ is the eccentricity. When one of the bodies is much greater than the other, as in the Sun - Mercury system, the latter equality is useful since it scales the result to the Schwarzschild radius of the primary body.

To calculate a numerical value for the relativistic perihelion shift $\Delta \phi$ for Mercury, substitute the Schwarzschild radius for the Sun, $r_{s}=2.96 \mathrm{~km}$, the semi-major axis of Mercury's orbit $a=57.91 \times 10^{6} \mathrm{~km}$, and its $e=0.2056$ into Equation (1.8). Multiply the result by 206,265 to go from radians to arcsec and by 415 , the number of revolutions per 100 years to obtain

$$
\Delta \phi=43^{\prime \prime}
$$

per century. The agreement found in this early test of General Relativity gave great confidence in the theory. Today's accuracy of testing General Relativity in the Solar System is at a level of $0.01 \%$. Here the characteristic velocity is $v / c \sim 10^{-4}$, and we are dealing with weak fields.

Another more extreme and recent test of General Relativity is the radio pulsar PSR $1913+16$, a smaller member of a binary system of two neutron stars about 1/60 as far 
from one another as Mercury is from the Sun. Using the semi-major axis of the binary $a \sim 1.95 \times 10^{6} \mathrm{~km}$, the total mass $M \sim 2.83 M_{\odot}$ and eccentricity $e \sim 0.617$, we find that in one year (1131 orbital revolutions) the periastron advances

$$
\Delta \phi \sim 4.2^{\circ} .
$$

The orbital parameters result in a prediction for the rate of loss of the orbital energy by gravitational radiation. It matches observations with the accuracy of $0.2 \%$ (Hulse and Taylor 1975, Taylor and Weisberg 1989, Damour and Taylor 1991, Hulse 1994). In binary pulsars, where the most interesting object is the double pulsar PRS J07373039 with the perihelion advance of $16.9^{\circ}$ per year (Lyne et al. 2004, Kramer et al. 2006), we have $v / c \sim 2 \times 10^{-3}$. Here we are also testing General Relativity in weak fields, but including gravitational radiation.

The dragging of space around rotating bodies in General Relativity was proposed by Austrian physicists Joseph Lense and Hans Thirring (1918). By 2004 the LenseThirring effect was measured in space surrounding the rotating Earth. Following the motions of two Earth satellites LAGEOS I and II, a team lead by Ignazio Cuifolini of University of Lecce, Italy, and Erricos Pavlis of University of Maryland, found that the planes of the orbits of the satellites have shifted by about two meters per year in the direction of the Earth's rotation due to dragging of space around the Earth (Cuifolini and Pavlis 2004). The result is in agreement with the prediction of Lense and Thirring within the $10 \%$ accuracy of the experiment. The satellite Gravity Probe B, designed for the measurement of space dragging confirmed these results in 2007 (Everitt et al. 2011). The Gravity Probe B Lens-Thirring value is $0.04^{\prime \prime} / \mathrm{yr}$ in contrast to the much larger $6.6^{\prime \prime} / \mathrm{yr}$ for the geodetic precession of its 7,027 km, 97.65 minute orbit around the Earth.

Relativistic spin-orbit coupling may cause the PSR 1913+16 pulsar's spin axis to precess (Damour and Ruffini 1974; Barker and O'Connell 1975a,b, Weisberg and Taylor 2005, Weisberg et al. 2010). This precession can result in changes in pulse shape as the pulsar-observer geometry changes. Under the assumption that relativistic precession is occurring, these changes have been used to model the two-dimensional structure of the pulsar beam.

Another early convincing test of the General Theory of Relativity is the bending of light rays which pass close to the Sun. Consider a photon passing by a mass $M$ with $b$ as the minimum distance between the photon and the mass $M$. The total deflection from a straight line path is (Einstein 1911)

$$
\Delta \phi=\frac{4 G M}{c^{2} b}=2 \frac{r_{s}}{b}
$$

radians. For a light ray passing the surface of the Sun, $r_{s}=2.96 \mathrm{~km}$ and $b=6.96 \times 10^{5}$ $\mathrm{km}$. General Relativity gives

$$
\Delta \phi=1.75^{\prime \prime}
$$


in good agreement with observations. Today better measurements using radio sources agree with Einstein's theory within $1 \%$ accuracy. A classical Newtonian calculation only gives half the relativistic value. Bending of light provided the second early convincing test of the General Relativity theory (Eddington 1919, Dyson et al. 1920). Via the deflection, stars close to the edge of the Sun appear to shift radially outward from the center of the Sun during a solar eclipse compared to a photograph of the same area six months earlier.

In a remarkable extension of the classical test of relativity beyond using the Sun, deflection of light from background sources has been seen around massive objects resulting in gravitational lensing, multiple images or strong distortions of the sources. The apparent source flux may also change. Multiple images of the quasar Q0957+561 were detected in 1979 by a team led by Dennis Walsh of the University of Manchester (Walsh et al. 1979) using the 2.1 meter telescope of Kitt Peak National Observatory. Nowadays gravitational lenses are detected frequently, and are used in astrophysical studies, in particular in estimating the relative amounts of dark matter in clusters of galaxies and matter's importance relative to dark energy in the universe.

One of the phenomena related to the elasticity of space is gravitational waves, small changes in the curvature of space which propagate in space with speed of light. At the moment, evidence for gravitational waves is indirect. The binary neutron star system PSR 1913+16 appears to emit gravitational waves. Observations show that the binary system does lose energy which cannot be explained in other ways beside gravitational wave emission. The loss rate of energy matches rather well what is expected in the General Relativity. This coincidence is usually taken as proof that gravitational waves do exist, even though the radiation from PSR 1913+16 is not presently directly measurable by gravitational wave antennas (Weisberg and Taylor 2005).

A promising case for both direct detection of gravitational radiation and the study of relativistic spin-orbit coupling is the binary black hole system of quasar OJ287 to be discussed later (Valtonen and Lehto 1997). Here one of the members is more massive than a star by a factor of $10^{10}$. Thus gravitational wave emission from this source should be very much more powerful than from the neutron stars of PSR 1913+16. The next generation of gravitational wave antennas should be able to directly confirm the emission of gravitational waves (Sun et al. 2011, Liu et al. 2012).

The curvature of spacetime around a rotating black hole was first calculated by the New Zealand mathematician Roy Kerr (1963). By conservation of angular momentum, a black hole arising from a rotating body must also rotate. The rotation of the black hole influences the surrounding spacetime even well beyond the black hole's Schwarzschild radius.

The quadrupole moment of the spinning primary black hole in OJ287 has a measurable effect on the orbit of the secondary. In OJ287, $v / c \sim 0.25$. Thus here we are carrying out strong field tests of General Relativity. For example, it has been shown that the loss of orbital energy from the system agrees with General Relativity with the accuracy of $2 \%$ (Valtonen et al. 2010b). More importatly, we may test the no hair 
theorems of black holes (Israel 1967, 1968, Carter 1970, Hawking 1971, 1972, see also Misner, Thorne and Wheeler 1973) for the first time. They relate the spin and the mass of a black hole to its quadrupole moment in a unique way (see Section 5).

Based on General Relativity, Einstein (1917) proposed a model for a curved finite (but still boundless) universe. The cosmological constant, $\Lambda$, was specified so as to produce a static universe with no origin in time. The evolving models of the universe, standard today, were derived by the Russian Alexander Friedmann in two papers in 1922 and 1924. These papers, a turning point in the study of cosmology, remained almost unnoticed. In 1927 Belgian astronomer Georges Lemaître rediscovered these models, now known as Friedmann universes.

Friedmann found a solution of the General Relativity equations which is more general than Einstein's solution. The Friedmann solution includes the Einstein static solution as a special case, where the cosmological constant is non-zero and related to the matter density so as to attain the gravity-antigravity balance. However, generally, the solution puts no restrictions on the cosmological constant. It might be zero or nonzero, positive or negative, related or unrelated to the matter density. The solution depends explicitly on time. The universe is not static: it expands or contracts as a whole.

Friedmann preferred expansion over contraction citing observational evidence supporting his choice in Slipher's data of galaxies that are moving away from us. Friedmann died in 1925 at the age of 37 before the Hubble discovery of the redshift distance relation (Hubble 1929). We can thus regard the discovery of the Hubble expansion as a predictive verification of General Relativity.

After the discovery of the expansion of the universe, and other evidence for an origin in time, the so called Big Bang, it became obvious that $\Lambda$ could not be as big as Einstein had calculated for his static universe model. It became common to assume that $\Lambda=0$. The ideas changed in the late 1990s when it became possible to use extremely luminous standard candles, supernovae of type Ia, to estimate distances of galaxies whose redshifts $z$ are comparable to unity. In 1998-99 two groups discovered the non-zero $\Lambda$, usually interpreted as an indication of cosmic vacuum or dark energy (Riess et al. 1998 and Perlmutter et al. 1999).

In General Relativity $\Lambda$ is a constant, but it could be imagined in other theories that $\Lambda$ would depend on cosmic time. This is something that we can test at the weak field limit of General Relativity. Local studies of $\Lambda$, if they show that it has the same value which is observed using distant supernovae, can in principle exclude many alternative models. This will be discussed in the following sections.

\section{Weak limit: concept of zero gravity surface}

In recent years it has become customary to move the $\Lambda$ term from the left hand side of Equation (1.1) to the right hand side. Then it may be viewed as a contribution to the energy momentum tensor. The corresponding density is called the vacuum density 
$\rho_{V}$, with the equation of state

$$
\rho_{V}=-P / c^{2}
$$

where $P$ is the vacuum pressure. The value of the vacuum density is related to $\Lambda$ by

$$
\Lambda=\frac{8 \pi G}{c^{2}} \rho_{V}
$$

Instead of $\Lambda$ it is common to state its normalized value

$$
\Omega_{\Lambda}=\frac{c^{2} \Lambda}{3 H_{0}^{2}}
$$

which is a dimensionless number of the order of unity. $H_{0}$ is the Hubble constant. From the cosmological recession of distant galaxies using Ia supernovae, the analysis of the microwave background radiation $(\mathrm{CMB})$ and by many other ways (see Section 5), we find

$$
\rho_{V} \approx 7 \times 10^{-30} \mathrm{gcm}^{-3}, \Omega_{\Lambda} \approx 0.73
$$

According to General Relativity, gravity depends on pressure as well as density: the effective gravitating density

$$
\rho_{e f f}=\rho+3 P / c^{2}
$$

It is negative for a vacuum: $\rho_{e f f}=-2 \rho_{V}$, and this leads to repulsion ("antigravity"). Hence the study of the antigravity in our neighbourhood, and on short scales in general, is an important test of General Relativity and its concept of Einstein's $\Lambda$-term in weak gravity conditions.

One way to study the local dark energy is via an outflow model (Chernin 2001; Chernin et al. 2006; Byrd et al. 2011) which describes expansion flows around local masses. It was motivated by the observed picture of the Local Group with outflowing dwarf galaxies around it (van den Bergh 1999; Karachentsev et al. 2009). The model treats the dwarfs as "test particles" moving in the force field produced by the gravitating mass of the group and the possible dark energy background. A static and spherically symmetric gravitational potential is a rather good approximation despite of the binary structure of the Local Group (Chernin et al. 2009).

\subsection{A gravitating system within dark energy and the zero-gravity radius}

Soon after the discovery of the universal acceleration from observations of distant supernovae, researchers returned to the old question of Järnefelt, Einstein and Straus about what happens to the spacetime around a mass concentration in an expanding universe and asked: at what distance from the Local Group do the gravity of its mass 
and the antigravity of the dark energy balance each other (Chernin 2001, Baryshev et al. 2001)?

Treating a mass concentration as a point mass $M$ on the background of the antigraviting dark energy, its gravity produces the radial force $-G M / r^{2}$, where $r$ is the distance from the group barycenter. The antigravity of the vacuum produces the radial force $G 2 \rho_{V}(4 \pi / 3) r^{3} / r^{2}=(8 \pi / 3) \rho_{V} r$. Here $-2 \rho_{V}$ is the effective gravitating density of vacuum. Then the radial component of motion in this gravity/antigravity force field obeys the Newtonian equation

$$
\ddot{r}=-G M / r^{2}+r / A^{2},
$$

where $r$ is the distance of a particle to the barycenter of the mass concentration. The constant

$$
A=\left[(8 \pi G / 3) \rho_{V}\right]^{-1 / 2}
$$

is the characteristic vacuum time and has the value $=5 \times 10^{17} \mathrm{~s}$ (or $16 \mathrm{Gyr}$ ) for $\rho_{V}=7 \times 10^{-30} \mathrm{~g} \mathrm{~cm}^{-3}$.

Equation (2.6) shows that the gravity force $\left(\propto 1 / r^{2}\right)$ dominates the antigravity force $(\propto r)$ at short distances. At the "zero-gravity distance"

$$
R_{V}=\left(G M A^{2}\right)^{1 / 3}=\left[(3 / 8 \pi)\left(M / \rho_{V}\right)\right]
$$

the gravity and antigravity balance each other, and the acceleration is zero. At larger distances, $r>R_{V}$, antigravity dominates, and the acceleration is positive. For the Local Group mass $M \approx 4 \times 10^{12} M_{\odot}$ and the global dark energy density, the zerogravity distance is $R_{V} \approx 1.6 \mathrm{Mpc}$.

Equation (1.4) shows that we arrive at the same concept also in full General Relativity.

If we try to calculate the zero-gravity radius around a point in a homogenous distribution, this radius will increase directly proportional to the radius of the considered matter sphere. So, one cannot ascribe physical significance to the zero-gravity radius within a fully uniform universe. But this is not true once some structure appears in the universe. A density enhancement does not appear alone, but together with the zero-gravity sphere around it.

The zero-gravity sphere for a point mass $M$ has special significance in an expanding universe. A light test particle at $r>R_{V}$ experiences an acceleration outwards relative to the point mass. If it has even a small recession velocity away from $M$, it participates in an accelerated expansion.

For an isolated system of two identical point masses $M$, the zero-gravity distance, where the two masses have zero-acceleration relative to their center-of-mass, is about $1.26 R_{V}$ (Teerikorpi et al. 2005). If separated by a larger distance, the masses will experience outward acceleration. 


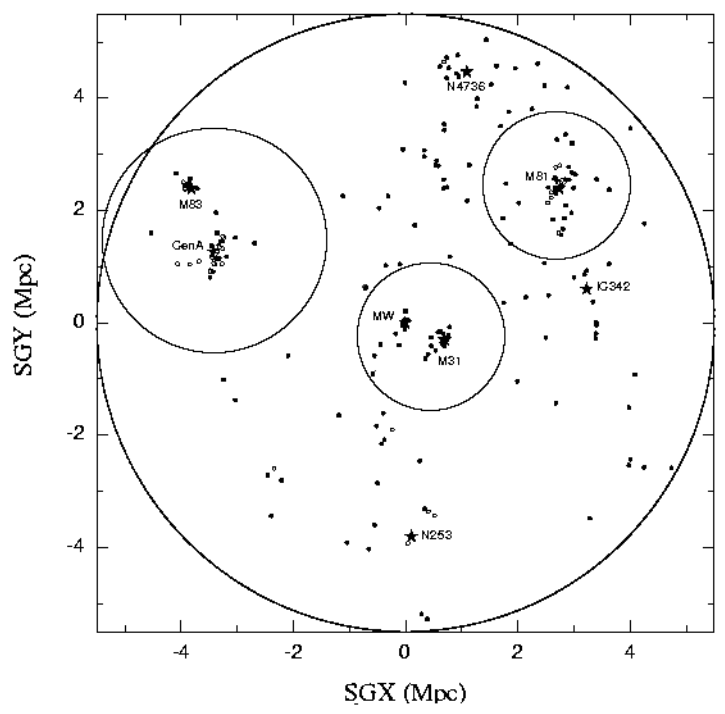

Figure 1. Approximate zero-gravity spheres around the Local Group (at the center) and two nearby galaxy groups. The radii have been calculated using the masses $2 \times 10^{12} M_{\odot}$ for the LG and the M81/M82 Group and $7 \times 10^{12} M_{\odot}$ for the CenA/M83 Group (the underlying map presents the local environment up to about $5 \mathrm{Mpc}$ as projected onto the supergalactic plane is from Karachentsev et al. 2003).

These examples illustrate the general result that in vacuum-dominated expanding regions perturbations do not grow. They also lead one to consider the Einstein-Straus (1945) solution where any local region may be described as a spherical expanding "vacuole" embedded within the uniform distribution of matter (Chernin et al. 2006).

The metric inside the vacuole is static and the zero-gravity radius and the (presenttime) Einstein-Straus vacuole radius are simply related:

$$
R_{E S}\left(t_{0}\right)=\left(2 \rho_{V} / \rho_{M}\right)^{1 / 3} R_{V}
$$

For instance, for the ratio $\rho_{V} / \rho_{M}=0.7 / 0.3$ one obtains $R_{E S}\left(t_{0}\right)=1.67 R_{V}$. The Einstein-Straus vacuole can be seen as the volume from which gravitation has gathered the matter to form the central mass concentration.

Figure 1 shows the map of our local extragalactic environment up to about $5 \mathrm{Mpc}$, together with the approximate zero-gravity spheres drawn around the Local Group and two nearby galaxy groups. The spheres do not intersect. This suggests that the groups are presently receding from each other with acceleration.

Using Eq. 2.8 one may calculate typical zero-gravity radii for different astronomical systems, for the standard value of $\rho_{V}$. For stars, star clusters, galaxies and tight binary galaxies the zero-gravity radius is much larger than the size of the system which is 
located deep in the gravity-dominated region. For galaxy groups and clusters, $R_{V}$ is near or within the region where the outflow of galaxies begins to be observed (about $1.6 \mathrm{Mpc}$ for the Local Group). It is especially on such scales where the system and its close neighborhood could shed light on the local density of dark energy. One is led to ask what happens to the test particles (dwarf galaxies) that have left the central region of the system?

\subsection{Dynamical structure of a gravitating system within dark energy}

The particles move radially practically as predicted by the Newtonian equation of motion (Eq. 2.6), where the forces are the gravity of the central mass and the antigravity of the dark energy. The first integral of this equation expresses the mechanical energy conservation:

$$
\frac{1}{2} \dot{r}^{2}=E-U(r)
$$

where $E$ is the total mechanical energy of a particle (per unit mass) and $U(r)$ is the potential energy

$$
U(r)=-\frac{G M}{r}-\frac{1}{2}\left(\frac{r}{A}\right)^{2}
$$

Because of the vacuum, the trajectories with $E<0$ are not necessarily finite. Such behavior of the potential has a clear analogue in General Relativity applied to the same problem.

The total energy of a particle that has escaped from the gravity potential well of the system must exceed the maximal value of the potential:

$$
E>U_{\max }=-\frac{3}{2} \frac{G M}{R_{\mathrm{V}}} .
$$

It is convenient to normalize the equations to the zero-gravity distance $R_{\mathrm{V}}$ and consider the Hubble diagram with normalized distance and velocity: $x=r / R_{\mathrm{V}}$ and $y=\left(V / H_{\mathrm{V}}\right) R_{\mathrm{V}}$ where $V$ is the radial velocity and $H_{\mathrm{V}}$ is a constant to be discussed below (Teerikorpi et al. 2008). Then radially moving test particles will move along curves which depend only on the constant total mechanical energy $E$ of the particle:

$$
y=x\left(1+2 x^{-3}-2 \alpha x^{-2}\right)^{1 / 2} .
$$

Here $\alpha$ parameterizes the energy, so that $E=-\alpha G M / R_{\mathrm{V}}$. Each curve has a velocity minimum at $x=1$, i.e. at $r=R_{\mathrm{V}}$.

The energy with $\alpha=3 / 2$ is the minimum energy which still allows a particle initially below $x=1$ to reach this zero-gravity border (and if the energy is slightly larger) to continue to the vacuum-dominated region $x>1$, where it starts accelerating. In the ideal case one does not expect particles with $x>1$ below this minimum velocity curve. 

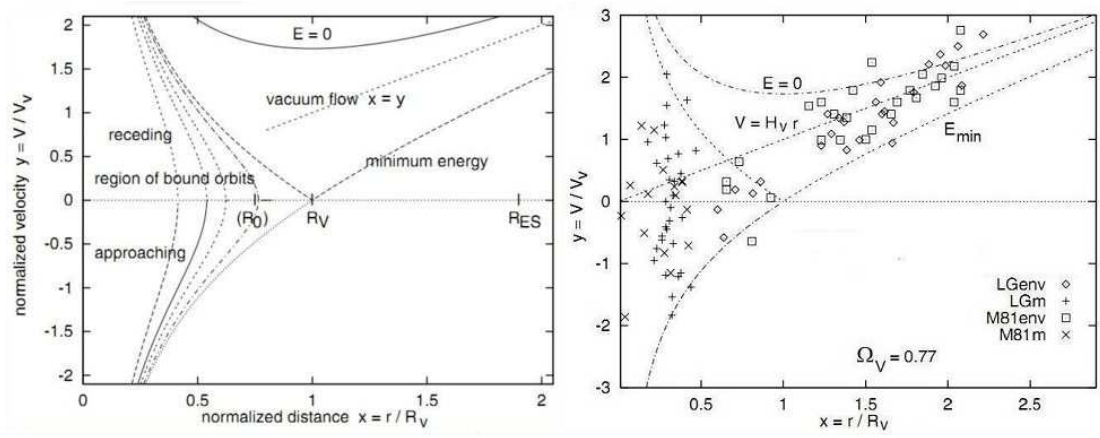

Figure 2. Left panel: Different regions in the normalized Hubble diagram around a point mass in the vacuum. In the region of bound orbits a dwarf galaxy cannot move into the vacuum flow region unless it receives extra energy as a result of an interaction with other galaxies. Right panel: The normalized Hubble diagram for the galaxies in the environments of the LG and M 81 groups, for the near standard vacuum density. The velocity-distance relation for the vacuum flow is shown. The curve for the lower limit velocity is given below and above $x=1$; below $x=1$ its negative counterpart is shown. The members of the groups, refered to by " $\mathrm{m}$ " in the symbol list, are found within these curves. (Adapted from Teerikorpi et al. 2008). 
Figure 2 (left panel) shows different regions in the normalized Hubble diagram. Below $r=R_{V}$, we have indicated the positive minimum velocity curve and its negative symmetric counterpart. This region defines the bound group: a galaxy will not escape beyond $R_{V}$ unless it obtains sufficient energy from an interaction.

The diagonal line $x=y$ gives the "vacuum" flow with the Hubble constant $H_{V}$, when dark energy is fully dominating. It is asymptotically approached by the outflying particles beyond $x=1$. This limit is described by de Sitter's static solution which has the metric of Eq. (1.2) with $M=0$. The spacetime of de Sitter's solution is determined by the vacuum alone, which is always static. It leads to the linear velocity-distance law, $V=r / A=H_{V} r$, with the constant expansion rate $H_{V}=\left(8 \pi G \rho_{V} / 3\right)^{1 / 2}$ :

$$
H_{V}=61 \times\left(\frac{\rho_{V}}{7 \times 10^{-30} \mathrm{~g} / \mathrm{cm}^{3}}\right)^{1 / 2} \mathrm{~km} / \mathrm{s} / M p c .
$$

The normalized vacuum energy density depends on $H_{\mathrm{V}}: \Omega_{\Lambda}=H_{\mathrm{V}}^{2} / H_{0}^{2}$. The vacuum Hubble time $T_{V}=1 / H_{\mathrm{V}}$ exceeds the global Hubble time $\left(=1 / H_{0}\right)$ by the factor $\left(1+\rho_{M} / \rho_{V}\right)^{1 / 2}=\left(\Omega_{\Lambda}\right)^{-1 / 2}$ for a flat universe. In the standard model $T_{V}=16 \times 10^{9}$ $\mathrm{yr}$ and the age of the universe $\left(13.7 \times 10^{9} \mathrm{yr}\right)$ is about $0.85 T_{V}$.

Figure 2 (right panel) shows a combined normalized Hubble diagram for the Local Group, the nearby M81 group, and their environments. The M81 group is at a distance of about $4 \mathrm{Mpc}$. We have used in this diagram the mass $2 \times 10^{12} M_{\odot}$ for both groups. The energy condition to overcome the potential well $\left(E>-3 / 2 G M / R_{\mathrm{V}}\right)$ is not violated in the relevant range $x=r / R_{\mathrm{V}}>1$.

\section{On local detection of dark energy: the Local Group}

One expects inward acceleration at distances $r<R_{V}$ and outward acceleration at distances $r>R_{V}$ within the region where the point-mass model is adequate. However, such accelerations in the nearby velocity field around the Local Goup are very small (of the order of $0.001 \mathrm{~cm} / \mathrm{s} / \mathrm{yr}$ ) and impossible to measure directly. We also cannot follow a dwarf galaxy in its trajectory for millions of years in order to see the location of the minimum velocity which defines theSeta zero-gravity distance.

The objects were likely expelled in the distant past within a rather narrow time interval (e.g., Chernin et al. 2004). What we see now is a locus of points at different distances from the center and lying on different energy curves; they make the observed velocity-distance relation.

\subsection{The present-day local Hubble flow}

The prediction for the present-day outflow of dwarf galaxies near the Local Group (or other galaxy systems) depends on the mass of the group, the flight time ( $<$ the age of the universe), and the local dark energy density. 

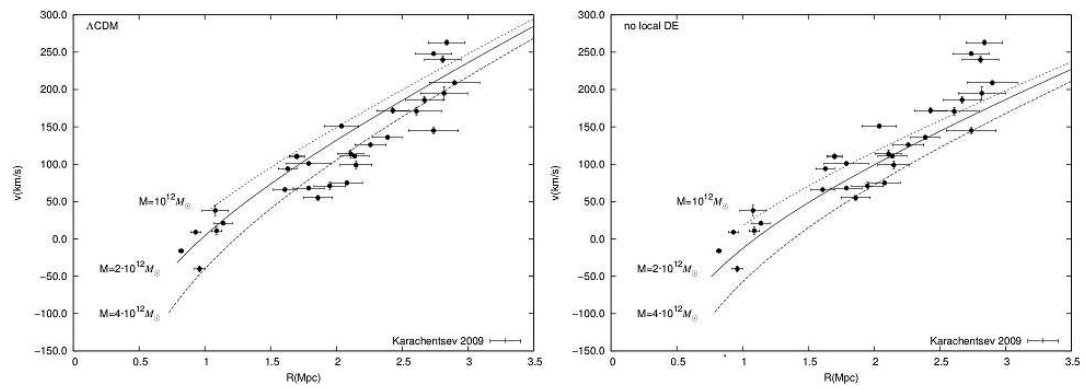

Figure 3. Left panel: The location of test particles as injected from the mass centre (curves) after the flight time $T_{0}=13.7 \mathrm{Gyr}$, for different masses $\left(1 \times 10^{12} M_{\odot}, 2 \times\right.$ $10^{12} M_{\odot}, 4 \times 10^{12} M_{\odot}$ ). Here the standard model is used (local dark energy = global dark energy). Right panel: The location of test particles after the flight time $T_{0}=13.7 \mathrm{Gyr}$, for different masses. Here the Swiss cheese model is used (no local dark energy). The data points are for the Local Group. (Credit. J. Saarinen)

Peirani \& de Freitas Pacheco (2008) derived the velocity-distance relation using the Lemaître-Tolman model containing the cosmological constant, and compared this with the model for $\Lambda=0$, using the central mass and the Hubble constant as free parameters. Chernin et al. (2009) considered the total energy for different outflow velocities at a fixed distance, and then calculated the required time for the test particle to fly from near the group's center up to this distance. The locus of the points corresponding to a constant age ( $\approx$ the age of the Universe) gave the expected relation.

Here we show some results from a modification of the above methods which easily allows one to vary the values of the relevant parameters and to generalize the model in various ways (Saarinen and Teerikorpi 2014). One generates particles close to the center of mass of the group and gives them a distribution of speeds. Then they are allowed to fly along the radial direction for a time $T$, and their distances from the center and velocities are noted. The flight time $T$, during which the integration of the equations of motion is performed, is at most the age of the standard Universe, 13.7 Gyr.

When comparing the standard $\Lambda \mathrm{CDM}$ model, with its constant dark energy density on all scales, it is relevant to consider the "Swiss cheese ( $\mathrm{SwCh}$ ) model", where the Universe has the same age as the standard model, but where the dark energy density is zero on small scales. This model could correspond to the case where dark energy (or analogous effects) operates on large scales only.

We plot some results together with the data on the local outflow around the Local Group from the Hubble diagram as derived by Karachentsev et al. (2009) from HST observations. The largest available distance is $3 \mathrm{Mpc}$.

Figure 3 shows the predicted distance-velocity curves for different masses in the standard model (left panel) and for the SwCh model (right panel). It is seen that the location of the curve depends rather strongly on the adopted mass. For instance, the 
SwCh curve with $M=2 \times 10^{12} M_{\odot}$ fits the LG data along the whole distance range. The $\Lambda$ CDM model requires $M \approx 4 \times 10^{12} M_{\odot}$ for a good fit beyond the zero-gravity distance.

Figure 3 shows the cases when the particles were ejected just after the Big Bang. This case would correspond to a classical situation where the outflow around the central group is "primordial". In practice, the age of the group and the outflowing dwarf galaxies must be less than the age of the Universe, and the origin of the outflow may be due to early interactions within the system, making galaxies escape from it (e.g., Valtonen et al. 1993, Byrd et al. 1994, Chernin et al. 2004). The dark energy antigravity enhances the escape probability because it makes the particle potential energy barrier lower than in the presence of gravity only.

The calculations show clear differences between the two cases in the sense expected: the $\Lambda \mathrm{CDM}$ curves are above the no-local-dark-energy curves and steeper, making the zero-velocity distance longer in the latter case, as already noted by Peirani \& de Freitas Pacheco (2008) and Chernin et al. (2009). However, in practice, the difference may be difficult to detect. First, the observed distance - velocity relation is rather scattered. Second, the independently known mass of the Local Group is uncertain. Thirdly, the model of the galaxy group and its evolution contains uncertain elements, including the exact ejection time.

\subsection{Mass, dark energy density and the "lost gravity" effect}

A general conclusion from the outflow data is that a low-mass Local Group ( $M \leq$ $2 \times 10^{12} M_{\odot}$ ) is associated with the case of no local dark energy, while a local density near the global dark energy density requires a higher mass, $M \approx 4 \times 10^{12} M_{\odot}$. This mutual dependence between the assumed mass and the derived dark energy density is typical for various local dynamical tests.

We may estimate conservative limits of local dark energy density $\rho_{l o c}$ as follows. If the value of $R_{\mathrm{V}}$ were known from the velocity-distance diagram and the mass $M$ of the group is independently measured, the dark energy density may be estimated in the outflow region:

$$
\frac{\rho_{l o c}}{\rho_{V}}=\left(\frac{M}{1.3 \times 10^{12} M_{\odot}}\right)\left(\frac{1.3 \mathrm{Mpc}}{R_{\mathrm{V}}}\right)^{3} .
$$

In fact, attempts to probe dark energy with nearby outflows were first made (e.g., Chernin et al. 2006, 2007; Teerikorpi et al. 2008) by using Equation (3.1). Also here the derived dark energy density depends directly on the assumed mass in addition to the strong inverse dependence on the used zero gravity distance.

The size of the group is a strict lower limit to $R_{V}$, giving an upper limit to the local dark energy density $\rho_{\mathrm{loc}}$, for a fixed group mass. Hence, for the Local Group $R_{V}>1$ Mpc leads to $\rho_{l o c}<2.2 \rho_{V}$, for the mass $M=2 \times 10^{12} M_{\odot}$.

An upper limit for $R_{V}$ would give the interesting lower limit to $\rho_{l o c}$. One way to study $R_{V}$ would be to find the distance $R_{E S}$, the Einstein-Straus radius where the local 


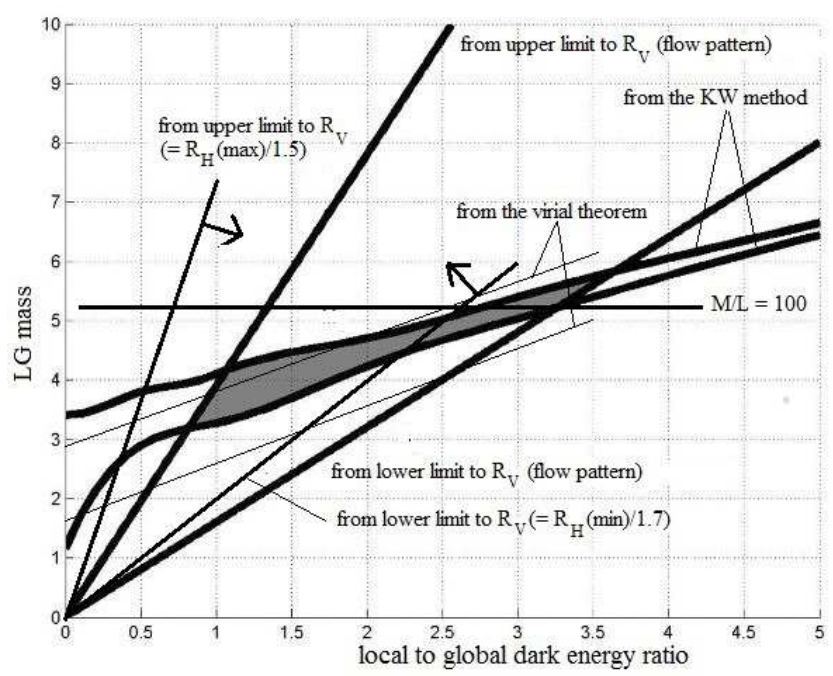

Figure 4. Results on the very local density of dark energy from the Local Group and its near environment (adapted from Chernin et al. (2009).

outflow reaches the global Hubble rate of Eq. (2.14) (Teerikorpi and Chernin 2010).

If there is no local dark energy, the outflow reaches the global expansion rate only asymptotically in this idealized point mass model. However, assuming that $\rho_{l o c}=\rho_{V}$, one may expect the local flow to intersect the global Hubble relation at a distance $R_{E S}=1.7 R_{V}$. For instance, with the Local Group mass of $(2-4) \times 10^{12} M_{\odot}$ and using the global dark energy density, one calculates $R_{E S}=1.7 R_{V}=2.2-2.6 \mathrm{Mpc}$. This range is indeed near the distance where the local expansion reaches the global rate (Figure 3; see also Karachentsev et al. 2009).

Starting with the illustrative value $R_{E S} \approx 2.6 \mathrm{Mpc}$, one may estimate that $R_{V} \approx$ 1.5 Mpc (Teerikorpi and Chernin 2010) and a lower limit for the dark energy density around the Local Group would be $\rho_{l o c} / \rho_{V} \geq 0.4$ for the mass of $2 \times 10^{12} M_{\odot}$. The limit is directly proportional to the adopted mass value.

In view of the uncertainties in using the outflow kinematics only, one should use in concert other independent methods for putting limits on the value of the local dark energy density. Such include the Kahn-Woltjer method and the virial theorem, both of which can be modified to take into account the "lost gravity" effect of dark energy ( Chernin et al. 2009).

Kahn and Woltjer (1959) used a simple one-dimensional two body problem to describe the relative motion of the Milky Way and M31 galaxies. The motion of the galaxies was described as a bound system. The (currently) observed values $r=0.7$ Mpc and $V=-120 \mathrm{~km} / \mathrm{s}$ lead to a limit for the estimated binary mass: $M>$ $1 \times 10^{12} M_{\odot}$. The mass is $M \approx 4.5 \times 10^{12} M_{\odot}$, if the maximum separation was about 4.4 Gyr ago, corresponding to $13.2 \mathrm{Gyr}$ as the time since the two galaxies started to 
separate from each other.

With a minimal modification of the original method, the first integral of the equation of motion (eq. (2.10)), in the presence of dark energy background, becomes:

$$
\frac{1}{2} V^{2}=\frac{G M}{r}+\left(\frac{G 4 \pi}{3}\right) \rho_{V} r^{2}+E .
$$

Now the total energy for a bound system embedded in the dark energy background must be smaller than an upper limit which depends on the mass and the dark energy density:

$$
E<-\frac{3}{2} G M^{3 / 2}\left[\left(\frac{8 \pi}{3}\right) \rho_{V}\right]^{1 / 3} .
$$

The limiting value corresponds to the case where the distance between the component galaxies could just reach the zero-gravity distance. Now the lower mass limit increases to $M>3.2 \times 10^{12} M_{\odot}$. Also the timing argument leads to an increased mass, $M \approx$ $5.3 \times 10^{12} M_{\odot}$ (Chernin et al. 2009; Binney \& Tremaine 2008). On the other hand, dynamical activity during the formation and settling down of the Local Group tends to reduce "timing" mass by a large factor (Valtonen et al. 1993). Though the uncertainty due to the various imperfectly known factors is rather large, the method suggests

$$
M \sim 4 \times 10^{12} M_{\odot}
$$

for the Local Group. A local volume cosmological simulation with this mass value agrees nicely with observations (Garrison-Kimmel et al. 2013).

The classical virial theorem is a well-known way to determine the mass of a quasistationary gravitationally bound many-particle system. For a system within dark energy, the virial theorem needs an extra term due to the contribution of the particle-dark energy interaction to the total potential energy (Forman 1970; Jackson 1970). When positive, the cosmological constant leads to a correction upwards for the mass estimates (Chernin et al. 2009) and one can show that the modified virial theorem should be written, in terms of the total mass $M$, a characteristic velocity $V$ and a characteristic sizes $R$, as:

$$
M=V^{2} \frac{R}{G}+\frac{8 \pi}{3} \rho_{V} R^{3},
$$

where the second term on the right hand side is the correction due to the dark energy. It is interestingly equal to the value of the effective (anti)gravitating mass of dark energy within the sphere of radius $R$. It is a measure of the lost-gravity effect, which can be significant in galaxy groups. In the Local Group the correction term contributes about 30 percent of the total mass.

Chernin et al. (2009) used the modified Kahn-Woltjer method together with the outflow data to derive the mass of the Local Group and the local dark energy density, resulting in $M=(3-6) \times 10^{12} M_{\odot}$ and $\rho_{l o c} / \rho_{V}=0.8-3.8$. The virial estimator which uses the mass and the velocity dispersion within the Local Group (Chernin et al. 2012) gave $\rho_{l o c} / \rho_{V}=0.7-2.8$. 
We present various Local Group results in Fig.4 where the horizontal axis gives the assumed local-to-global dark energy density and the vertical axis is the mass of the Local Group. A simpler version of this diagram appeared in Chernin et al. (2009), where the admissible range from the modified Kahn-Woltjer -method was shown, together with the limiting straight lines corresponding to the upper and lower limit of $R_{V}$ as estimated from the appearance of the outflow pattern. These defined the darkened area as the possible range of the mass and the local dark energy density. The present Fig.4 includes additional constraints:

1) The range of the virial mass based on van den Bergh's (1999) result $M_{\mathrm{LG}}=$ $2.3 \pm 0.6 M_{\odot}$ and using the correction term in the appendix to Chernin et al. (2009).

2) The location of the mass-to-luminosity ratio $M / L=100$ (an upper limit for small groups) as the horizontal line, based on van den Bergh's (1999) result that $M_{\mathrm{LG}}=2.3 \times 10^{12} M_{\odot}$ corresponds to $M / L=44$, and

3 ) The lines corresponding to upper and lower limits to $R_{V}$ as inferred from the distance range $2.2-3 \mathrm{Mpc}$ where the local flow reaches the global Hubble rate and using the calculations in Teerikorpi \& Chernin (2010). These limits may be less subjective than the original thick lines which were based on visual inspection of the flow pattern.

We see that the different constraints from the Local Group put the local dark energy density into the range $\rho_{l o c} / \rho_{V}=0.5-2.5$.

The preceding discussion did not make any use of the prior knowledge of the dwarf galaxy outflow. Dwarf galaxies are thrown out of the Local Group during its early assembly and later evolution. In cosmological N-body simulations where this process is seen, it is sometimes called a "back splash". The "back splash" follows the normal rules of dynamical ejection in a potential well (Valtonen and Karttunen 2006). The dwarf ejection times have a wide distribution, with a higher rate in the beginning. However, since the Local Group is dynamically young, as the two major galaxies have not yet completed even one full orbit, the dwarf ejection rate has not yet declined signicantly.

The distribution of the ejection speeds $P(V)$ is a steeply declining function of $V$. The probability that the escape velocity is in the interval $V, V+d V$ is

$$
P d V \sim V^{-4.5} d V
$$

(Valtonen and Karttunen 2006, Eq. (11.33)). What it means that in practice all dwarfs cross the zero gravity surface with a speed which is very close to zero $(\sim 40 \mathrm{~km} / \mathrm{s})$. It has two consequences: the flow speed of dwarfs beyond the surface is very smooth, and the time spent by the dwarfs at the zero gravity sphere is long and consequently we find an accumulation of the flow at this boundary. This makes the identification of the zero gravity radius simple. For the Local group

$$
R_{V}=1.6 \pm 0.1 M p c .
$$

The two main galaxies lose orbital energy from their relative motion every time a galaxy is ejected from the group. Therefore the Local Group timing argument (Kahn 
and Woltjer 1959), when applied to the isolated pair, gives necessarily an overestimate of their combined mass. The ejections shorten the major axis of the relative orbit, and thus less mass is required to close the orbit. The importance of this effect depends on the total mass of the ejected galaxies. In the scenarios calculated by Valtonen et al. (1993) the effect is 5\% in the universe of 14 billion yr in age. A greater reduction in the "timing mass", up to $25 \%$, comes from the possibility that the rotation speed of the Galaxy is greater than the standard $220 \mathrm{~km} / \mathrm{s}$. On the other hand, the lost gravity effect would tend to increase the "timing mass" by about $30 \%$.

Therefore, all in all, the full N-body simulation model of Garrison-Kimmel et al. (2013), with Local Group mass of $M=4 \times 10^{12}$ solar mass, appears to be an acceptable model of the Local Group and the surrounding flows. With these values we get $\Omega_{\Lambda}=0.75$, the value given on the first line of Table 1 (Section 5). The one standard deviation uncertainty may be estimated as $\pm 10 \%$.

Another way to obtain the local $\Omega_{\Lambda}$, is to take the measured values of $H_{V}$ and $H_{0}$ from observations, and calculate

$$
\Omega_{\Lambda}=\left(\frac{H_{V}}{H_{0}}\right)^{2}
$$

Even though both quantities on the right hand side have associated uncertainties, we get using $H_{V}=59 \mathrm{~km} / \mathrm{s} / \mathrm{Mpc}$ and $H_{0}=70 \mathrm{~km} / \mathrm{s} / \mathrm{Mpc}$, an estimate $\Omega_{\Lambda}=0.71$ (Chernin 2013), again with the estimated one standard deviation uncertainty of $\pm 10 \%$. This value is given on the second line of Table 1.

\section{Dark energy in the Coma cluster of galaxies}

In this Section, we extend our studies of the local dark energy effects from groups of galaxies to clusters of galaxies and address the Coma cluster considering it as the largest regular, nearly spherically-symmetrical, quasi-stationary gravitationally bound aggregation of dark matter and baryons embedded in the uniform background of dark energy. Is antigravity produced by dark energy significant in the volume of the cluster? Does it affect the structure of the cluster? Can antigravity put limits on the major gross parameters of the system? In a search for answers to these questions, we will use and develop the general considerations on the local dark energy given in the sections above.

\subsection{Three masses of the cluster}

The mass of the Coma cluster was first measured by Zwicky $(1933,1937)$ decades ago. Using the virial theorem he found that it was $3 \times 10^{14} M_{\odot}$ when normalized to the presently adopted value of the Hubble constant $H_{0}=70 \mathrm{~km} / \mathrm{s} / \mathrm{Mpc}$ which is used here. Later The \& White (1986) found an order of magnitude larger value, $2 \times 10^{15} M_{\odot}$, with a modified version of the virial theorem. Hughes $(1989,1998)$ obtained a similar value 
$(1-2) \times 10^{15} M_{\odot}$ with $\mathrm{X}$-ray data under the assumption that the hot intergalactic gas in the cluster is in hydrostatic equilibrium. With a similar assumption, Colless (2002) reports the mass $4.4 \times 10^{14} M_{\odot}$ inside the radius of $1.4 \mathrm{Mpc}$. A weak-lensing analysis gave the mass of $2.6 \times 10^{15} M_{\odot}$ (Kubo et al. 2007) within $4.8 \mathrm{Mpc}$ radius. Geller et al. $(1999,2011)$ examined the outskirts of the cluster with the use of the caustic technique (Diaferio \& Geller 1997, Diaferio 1999) and found the mass $2.4 \times 10^{15} M_{\odot}$ within the $14 \mathrm{Mpc}$ radius. Taken at face value, it appears that the mass within $14 \mathrm{Mpc}$ is smaller than the mass within $4.8 \mathrm{Mpc}$. Most probably, this is due to uncertainties in mass determination. Indeed, the $2 \sigma$ error is $1.2 \times 10^{15} M_{\odot}$ in Geller's et al. (1999, 2011) data, and within this uncertainty, the result does not contradict the small-radius data.

Also one should note that the action of the central binary in the Coma cluster has the effect of ejecting galaxies from the cluster. It leads to an overestimate of the cluster mass if virial theorem is used (Valtonen and Byrd 1979, Valtonen et al. 1985, Laine et al. 2004).

In each of the works mentioned here, the measured mass is treated as the matter (dark matter and baryons) mass of the cluster at various clustercentric distances. However, the presence of dark energy in the volume of the cluster modifies this treatment, since dark energy makes its specific contribution to the mass of the system. This contribution is naturally measured by effective gravitating mass of dark energy in the volume of the cluster at various clustercentric distances $R$ (Eq. 3.5):

$$
M_{V}(R)=\frac{4 \pi}{3} \rho_{V e f f} R^{3}=-\frac{8 \pi}{3} \rho_{V} R^{3}=-0.85 \times 10^{12}\left[\frac{R}{1 M p c}\right]^{3} M_{\odot} .
$$

For the largest radius $R=14 \mathrm{Mpc}$ we have:

$$
M_{V}=-2.3 \times 10^{15} M_{\odot}, \quad R=14 \mathrm{Mpc} .
$$

The total gravitating mass within the radius $R$ is the sum

$$
M_{G}(R)=M_{M}(R)+M_{V}(R),
$$

where $M_{M}(R)$ is the matter (dark matter and baryons) mass of the cluster inside the same radius $R$. It is this mass $M_{G}(R)$ that is only available for astronomical measurements via gravity (with virial, lensing, caustic, etc. methods). Because of this, we identify the gravitating mass $M_{G}(R)$ with the observational masses quoted above for various clustrocentric radii. In particular, the gravitating mass for the largest radius $R=14 \mathrm{Mpc}$ in the Coma cluster is this:

$$
M_{G}(R)=M_{V}(R)+M_{M}(R)=2.4 \times 10^{15} M_{\odot} .
$$

Then the matter mass $M_{M}$ at the same radius

$$
M_{M}(R)=M_{G}(R)-M_{V}(R)=4.7 \times 10^{15} M_{\odot} .
$$


We see that the value of the matter mass $M_{M}$ at $R=14 \mathrm{Mpc}$ obtained with the presence of dark energy is a factor of (almost) two larger than that in the traditional treatment. This implies that the antigravity effects of dark energy are strong at large radii of the Coma cluster.

\subsection{Matter mass profile}

In the spherically symmetric approximation, the cluster matter mass $M_{M}(R)$ may be given in the form:

$$
M_{M}(R)=4 \pi \int \rho(R) R^{2} d R
$$

where $\rho(R)$ is the matter density at the radius $R$. According to the widely used NFW density profile (Navarro et al. 1997)

$$
\rho=\frac{4 \rho_{s}}{\frac{R}{R_{s}}\left(1+\frac{R}{R_{s}}\right)^{2}},
$$

where $\rho_{s}=\rho\left(R_{s}\right)$ and $R_{s}$ are constant parameters. At small radii, $R<<R_{s}$, the matter density goes to infinity, $\rho \propto 1 / R$ as $R$ goes to zero. At large distances, $R>>$ $R_{s}$, the density slope is $\rho \propto 1 / R^{3}$. With this profile, the matter mass profile is

$$
M_{M}(R)=16 \pi \rho_{s} R_{s}^{3}\left[\ln \left(1+R / R_{s}\right)-\frac{R / R_{s}}{1+R / R_{s}}\right]
$$

To find the parameters $\rho_{s}$ and $R_{s}$, we may use the small-radii data (as quated above): $M_{1}=4.4 \times 10^{14} M_{\odot}$ at $R_{1}=1.4 \mathrm{Mpc}, M_{2}=2.6 \times 10^{15} M_{\odot}$ at $R_{2}=4.8 \mathrm{Mpc}$. At these radii, the gravitating masses are practically equal to the matter masses there. The values of $M_{1}, R_{1}$ and $M_{2}, R_{2}$ lead to two logarithmic equations for the two parameters of the profile, which can easily be solved: $R_{s}=4.7 \mathrm{Mpc}, \rho_{s}=1.8 \times 10^{-28} \mathrm{~g} / \mathrm{cm}^{3}$. Then we find the matter mass within $R=14 \mathrm{Mpc}$,

$$
M_{M} \simeq 8.7 \times 10^{15} M_{\odot},
$$

to be considerably larger (over $70 \%$ ) than given by our estimation above.

Another popular density profile (Hernquist 1990) is

$$
\rho(R) \propto \frac{1}{R(R+\alpha)^{3}} .
$$

Its small-radius behavior is the same as in the NFW profile: $\rho \rightarrow \infty$, as $R$ goes to zero. The slope at large radii is different: $\rho \propto 1 / R^{4}$. The corresponding mass profile is

$$
M_{M}(R)=M_{0}\left[\frac{R}{R+\alpha}\right]^{2} .
$$


The parameters $M_{0}$ and $\alpha$ can be found from the same data as above on $M_{1}, R_{1}$ and $M_{2}, R_{2}: M_{0}=1.4 \times 10^{16} M_{\odot}, \alpha=6.4 \mathrm{Mpc}$, giving another value for the mass within $14 \mathrm{Mpc}$ :

$$
M_{M}=6.6 \times 10^{15} M_{\odot}, \quad R=14 \mathrm{Mpc} .
$$

Now the difference from our estimated figure is about $40 \%$.

In a search for a most suitable mass profile for the Coma cluster, we may try the following simple new relation:

$$
M_{M}(R)=M_{*}\left[\frac{R}{R+R_{*}}\right]^{3} .
$$

This mass profile comes from the density profile:

$$
\rho(R)=\frac{3}{4 \pi} M_{*} R_{*}\left(R+R_{*}\right)^{-4} .
$$

The density goes to a constant as $R$ goes to zero; at large radii, $\rho \propto 1 / R^{4}$, as in Hernquist's profile.

The parameters $M_{*}$ and $R_{*}$ are found again from the data for the radii of 1.4 and $4.8 \mathrm{Mpc}: M_{*}=8.7 \times 10^{15} M_{\odot}, R_{*}=2.4 \mathrm{Mpc}$. The new profile leads to a lower matter mass at $14 \mathrm{Mpc}$ :

$$
M_{M}=5.4 \times 10^{15} M_{\odot}, \quad R=14 M p c,
$$

which is equal to our estimate above within $15 \%$ accuracy.

\subsection{Upper limits and beyond}

It is obvious that a system of galaxies can be gravitationally bound only if gravity dominates over antigravity in its volume. In terms of the characteristic masses introduced above, this condition may be given in the form:

$$
M_{M}(R) \geq\left|M_{\mathrm{V}}(R)\right|
$$

The condition is naturally met in the interior of the system. But the dark energy mass $\left|M_{\mathrm{V}}(R)\right|$ increases with the radius as $R^{3}$, while the matter mass increases slower in all the three versions of the matter mass profile $M_{M}(R)$ discussed above. As a result, an absolute upper limit arises from this condition to the total size and the total matter mass of the cluster. For the new matter mass profile introduced above one has:

$$
R_{\max }=20 \mathrm{Mpc}, \quad M_{\max }=6.2 \times 10^{15} M_{\odot} .
$$

These upper limits are consistent with the theory of large-scale structure formation that claims the range $2 \times 10^{15}<M<10^{16} M_{\odot}$ for the most massive bound objects in the Universe (Holz \& Perlmutter 2012, Busha et al. 2005). 
For comparison, the two traditional matter profiles mentioned above lead to somewhat larger values of the size and mass:

$$
\begin{gathered}
R_{\max }=25 \mathrm{Mpc}, \quad M_{\max }=1.5 \times 10^{16} M_{\odot}(\mathrm{NFW}), \\
R_{\max }=22 \mathrm{Mpc}, \quad M_{\max }=9.1 \times 10^{15} M_{\odot}(\text { Hernquist }) .
\end{gathered}
$$

Generally, the limit condition $M_{G}\left(R_{\max }\right)=\left|M_{V}\left(R_{\max }\right)\right|$ leads to the relation between the upper mass limit and the upper size limit:

$$
R_{\max }=\left(\frac{3 M_{\max }}{8 \pi \rho_{V}}\right)^{1 / 3} .
$$

The relation shows that the upper size limit is identical to the zero-gravity radius introduced in Section 1: $R_{\max }=R_{\mathrm{V}}$.

Studies of nearby systems like the Local Group and the Virgo and Fornax clusters (Karachentsev et al. 2003, Chernin 2008, Chernin et al. 2003, 2007, 2010, 2012a,b, 2013, Chernin 2013, Hartwick 2011) show that their sizes are indeed near the corresponding zero-gravity radii, so that each of the systems occupies practically all the volume of its gravity-domination region $\left(R \leq R_{\mathrm{V}}\right)$. These examples suggest that the Coma cluster may have the maximal possible size and its total matter mass may be near the maximal possible value. If this is the case, the mean matter density in the system is expressed in terms of the dark energy density and does not depend on the density profile (Merafina et al. 2012; Bisnovatyi-Kogan and Chernin 2012, Bisnovatyi-Kogan and Merafina 2013):

$$
\left\langle\rho_{\mathrm{M}}\right\rangle=\frac{M_{M}}{\frac{4 \pi}{3} R_{\mathrm{V}}^{3}}=2 \rho_{\mathrm{V}} .
$$

Another theoretical prediction may be made about the environment of the Coma cluster at the dark energy domination area $R>R_{V}$. Earlier studies have shown that outflows of galaxies exist around the Local group and some other groups and clusters of galaxies. We may assume that such an outflow may be observed around the Coma cluster as well at the distance $R>R_{V} \simeq 20 \mathrm{Mpc}$ from its center. The outflow is expected to have quasi-regular kinematical structure with the nearly linear velocity-distance relation. The galaxies in the outflow have been ejected by dynamical interactions in the cluster center (Saarinen and Valtonen 1985, Byrd et al. 2007).

These theoretical predictions may be tested in current and future astronomical observations of the Coma cluster and its environment. The next generation of ground based and space telescopes should be able to resolve individual stars in the galaxies of the Coma cluster, and allow us to construct a three-dimensional map of the cluster together with the radial velocities of each galaxy. We may then be able to separate the "warm" inflow of galaxies in the outskirts of the cluster from the "cool" outflow, and to determine the position of the zero-gravity radius experimentally. 


\section{Testing the constancy of $\Lambda$}

The concept of dark energy (or more specifically Einstein's cosmological constant) has become a routine factor in global cosmology on Gpc scales. We have shown above that it is also relevant in the local extragalactic universe. In the local weak gravity conditions the antigravity effect of dark energy becomes measurable and has to be included in dynamical studies performed in galaxy group and cluster scales of a few Mpc. In general, extragalactic mass determinations should include a correction term due to the 'lost gravity' effect caused by dark energy. Here we briefly mention a few other applications.

The Hubble outflow is one factor in explaining the well-known redshift anomaly in local galaxy group data via a selection effect (Byrd and Valtonen 1985, Valtonen and Byrd 1986, Niemi and Valtonen 2009). This asymmetry between redshifts and blueshifts of group members can be seen as a signature of local dark energy (Byrd et al. 2011).

The zero-gravity radius is an important quantity, which has existed (with roughly the same value) since the formation of a mass concentration in the expanding universe. It defines a natural upper limit to the size of a gravitationally bound system, allowing one to give an upper limit to the cosmic gravitating matter density in the form of galaxy systems. For the local universe this was discussed by Chernin et al. (2012b).

The measurement of the local value of the density of dark energy is naturally very important for our understanding of the nature of dark energy. Is it really constant on all scales and at all times, as suggested by the original concept of Einstein's cosmological constant?

After the initial period of rediscovery of the cosmological constant $\Lambda$ in 1998-1999, there has been been a large amount of activity in trying to determine the exact value of the constant, and to study its possible dependence on redshift (Frieman et al. 2008, Blanchard 2010, Weinberg et al. 2013). As we stated before, in General Relativity $\Lambda$ is an absolute constant.

Tables 1-4 list a sample of $\Omega_{\Lambda}$ found in the literature from year 2005 onwards together with the one standard deviation error limits. The values cluster around $\Omega_{\Lambda}=$ 0.73 with a standard deviation of 0.044 . This is somewhat smaller than the typical error in individual measurements. Only the cosmic microwave background (CMB) models give a significantly better accuracy, but in this case one may suspect hidden systematic errors due to foreground corrections (Whitbourn et al. 2014). Within the error bounds, $\Omega_{\Lambda}$ is constant over the redshift range that can be studied. Note that the CMB model values have been placed at redshift $z=3$ since the higher redshift universe does not affect the derived $\Omega_{\Lambda}$ significantly. The $\Omega_{\Lambda}$ values obtained at the weak limit are the same as the cosmological determinations within errors. 


\section{Strong limit: Spinning black holes and no-hair theorem}

There is plenty of evidence in support of the existence of black holes having masses in the range from a few $M_{\odot}$ to a few $10^{10} M_{\odot}$ (see A. Fabian elsewhere in this book). However, to be sure that these objects are indeed the singularities predicted by General Relativity, we have to ascertain that at least in one case the spacetime around the suspected black hole satisfies the no-hair theorems. The black hole no-hair theorems state that an electrically neutral rotating black hole in GR is completely described by its mass $M$ and its angular momentum $S$. This implies that the multipole moments, required to specify the external metric of a black hole, are fully expressible in terms of $M$ and $S$. It is important to note that the no-hair theorems apply only in General Relativity, and thus they are a powerful discriminator between General Relativity and various alternative theories of gravitation that have been suggested (Will 2006, Yunes and Siemens 2013, Gair et al. 2013).

A practical test was suggested by Thorne and Hartle (1985) and Thorne et al. (1986). In this test the quadrupole moment $Q$ of the spinning body is measured. If the spin of the body is $S$ and its mass is $M$, we determine the value of $q$ in

$$
Q=-q \frac{S^{2}}{M c^{2}} .
$$

For true black holes $q=1$, and for neutron stars and other possible bosonic structures $q>2$ (Wex and Kopeikin 1999, Will 2008). In terms of the Kerr parameter $\chi$ and the dimensionless quadrupole parameter $q_{2}$ the same equation reads

$$
q_{2}=-q \chi^{2}
$$

where $q_{2}=c^{4} Q / G^{2} M^{3}$ and $\chi=c S / G M^{2}$.

An ideal test of the no-hair theorem is to have a test particle in orbit around a spinning black hole, and to follow its orbit. Fortunately, there exists such a system in nature. The BL Lacertae object OJ287 is a binary black hole of very large mass ratio, and it gives well defined signals during its orbit. These signals can be used to extract detailed information on the nature of the orbit, and in particular, to find the value of the parameter $q$ in this system.

The optical light curve of this quasar displays periodicities of 11.8 and 55 years (Sillanpää et al. 1988, Valtonen et al. 2006), as well as a 50-day period of outbursts (Pihajoki et al. 2013). A model which explains these cycles, as well as a wealth of other information on OJ287 is discussed in the next section.

One of the pieces of information that we are able to find out is the spin of the primary black hole, $\chi_{1}=0.25 \pm 0.04$. The timing of the next outburst at the beginning of 2016 should help to improve the accuracy of $\chi$ to about $\pm 5 \%$. The mass of the primary is already determined with the accuracy of $\pm 1 \%$ which means that at least in principle we could reach the accuracy of $\pm 10 \%$ in measuring $q$ (Valtonen et al. 2011a).

There exist a number of other proposals to test the black hole no-hair theorems. The scenarios include the radio timing of eccentric millisecond binary pulsars which 


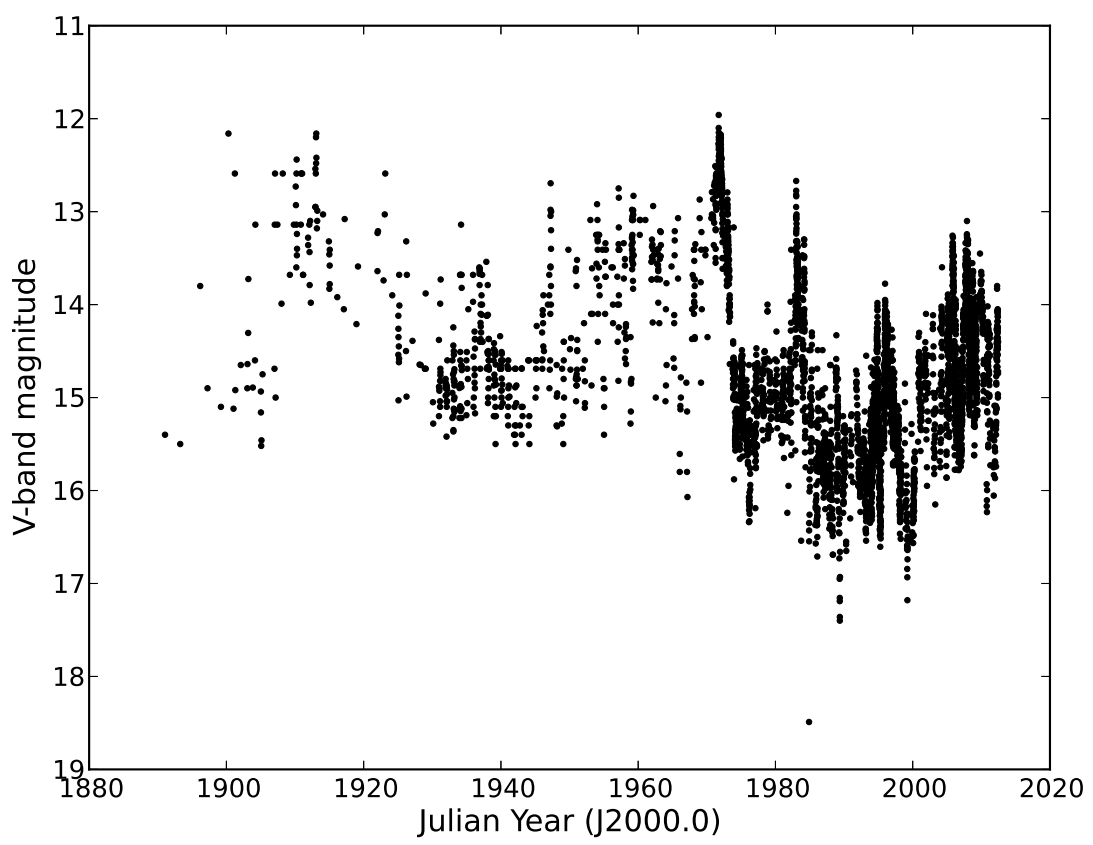

Figure 5. The observation of the brightness of OJ287 from late 1800's until today.

orbit an extreme Kerr black hole (Wex and Kopeikin 1999). Such systems are yet to be discovered. Also one may use several stars orbiting the massive Galactic center (Sgr A*) black hole at milliarcsec distances, if such stars are discovered and they are followed by infrared telescopes of the future, capable of doing astrometry at $\sim 10 \mu$ arcseconds level (Will 2008). Observations of gravitational waves from mergers of supermassive black holes, when they become possible some decades from now, may also be used to test the theorems (Barack and Cutler 2007).

The imaging of accretion flow around $\mathrm{Sgr} \mathrm{A}^{*}$, when it becomes possible, may allow the testing of no-hair theorems. The test relies on the argument that a bright emission ring characterizing the flow image will be elliptical and asymmetric if the theorems are violated (Broderick et al. 2013). Finally, quasiperiodic oscillations, relativistically broadened iron lines, continuum spectrum and X-ray polarization in the accretion disk surrounding a spinning black hole may also be used as a probe of the no-hair theorems (Johannsen and Psaltis 2010a, 2010b, 2011, 2013, Bambi and Barausse 2011a, 2011b, Bambi 2012a, 2012b, 2012c, 2013, Krawczynski 2012).

It is expected that some of these tests should be possible by the middle of the next decade. Generally, there may be some difficulty due to the potential degeneracy be- 
tween erraneous accretion physics close to the last stable orbit, which itself is not fully understood, and deviations from General Relativity (Broderick et al. 2013). In contrast, the test in $\mathrm{OJ} 287$ has already been done, and there are good prospects of improving the accuracy to $\pm 10 \%$ later in this decade. It does not depend on the accretion physics so close to the black hole. In what follows, we briefly summarize the current knowledge of the OJ287 system and the present constraints on the value of $q$.

\section{OJ287 binary system}

The identification of the OJ287 system as a likely binary was made already in 1980's, but since the mean period of the system is as long as $12 \mathrm{yr}$, it has taken a quarter of century to find convincing proof that we are indeed dealing with a binary system (Valtonen 2008, Valtonen et al. 2008a, 2008b). The primary evidence for a binary system comes from the optical light curve. By good fortune, the quasar OJ287 was photographed accidentally since 1890's, well before its discovery in 1968 as an extragalactic object. The pre-1968 observations are generally referred to as "historical" light curve points. The light curve of over one hundred years (Figure 5) shows a pair of outbursts at $\sim 12$ yr intervals. The two brightness peaks in a pair are separated by $1-2$ yrs.

The system is not strictly periodic, but there is a simple mathematical rule which gives all major outbursts of the optical light curve record. To define the rule, take a Keplerian orbit and demand that an outburst is produced at a constant phase angle and at the opposite phase angle. Due to the nature of Keplerian orbits, this rule cannot be written in a closed mathematical form, but the outburst times are easily calculated from it. According to this rule two outburst peaks arise per period. By choosing an optimal value of eccentricity (which turns out to be $e \sim 0.7$ ) and by allowing the semimajor axis of the orbital ellipse to precess in forward direction at an optimal rate (which turns out to be $\Delta \phi \sim 39^{\circ}$ per period), the whole historical and modern outburst record of $\mathrm{OJ} 287$ is well reproduced.

The type of model that follows from this rule is immediately obvious. It consists of a small black hole in orbit around a massive black hole. The secondary impacts the accretion disk of the primary twice during each full orbit (Figure 6). The two impacts produce the two flares that are observed 1 - 2 years apart, and the flares are repeated in every orbital cycle. However, because of relativistic precession, the pattern of flares is not exactly periodic. It is this fact that allows the determination of the precession rate, and from there the mass of the primary in a straightforward way. Note that it is not necessary to know the inclination of the secondary black hole orbit nor the orientation of the system relative to the observer in order to carry out these calculations.

The orbit that follows from the observed mathematical rule can be solved as soon as 5 flares are observed. Five flares have four intervals of time as input parameters, and the solution gives four parameters of the orbit uniquely: primary mass $m_{1}$, orbit eccentrity $e$, precession rate $\Delta \phi$, and the phase angle $\phi_{0}$ at a given moment of time. There does not need to be any solution at all if the basic model is not correct. However, 


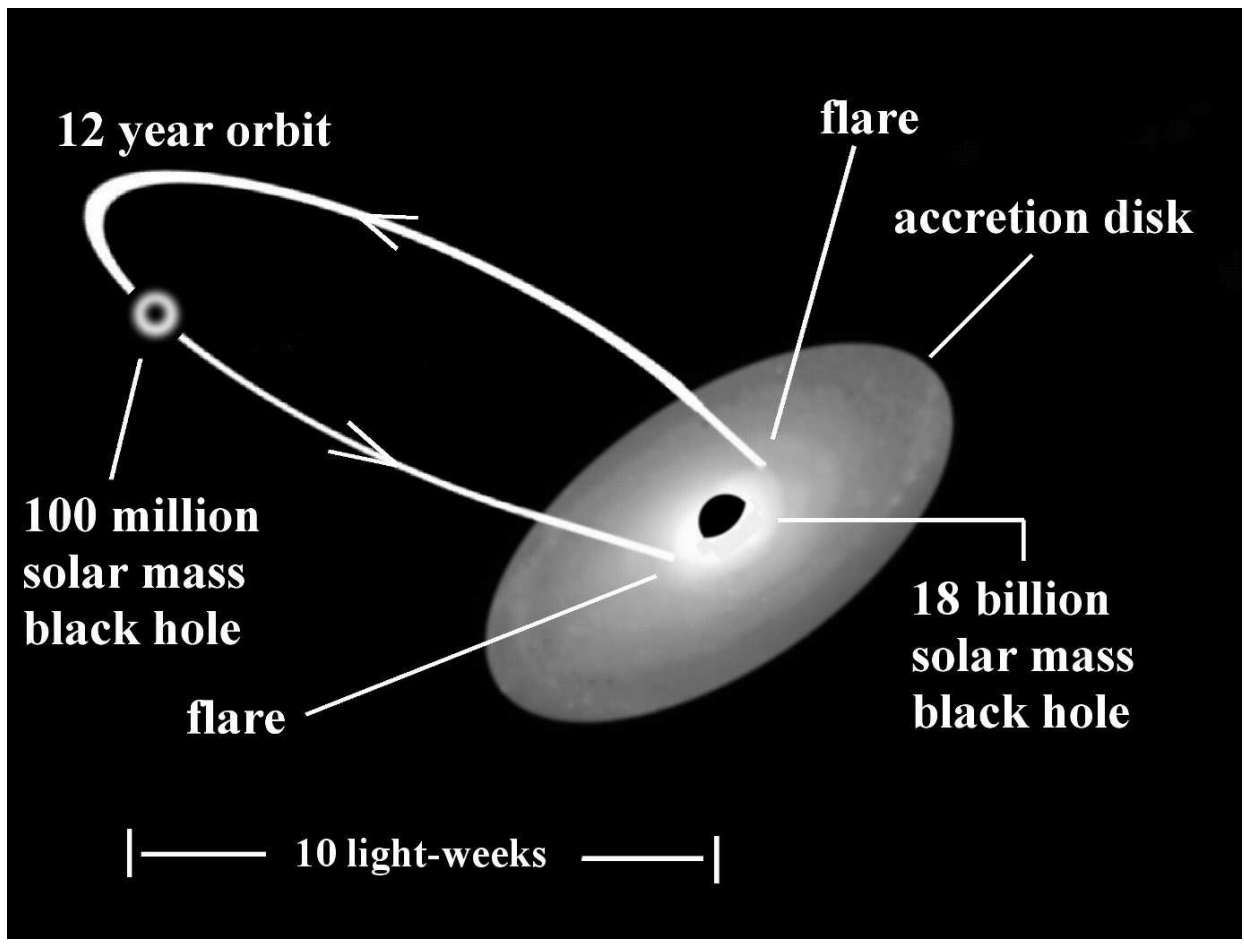

Figure 6. An illustration of the OJ287 binary system. The jets are not shown, but they may be taken to lie along the rotation axis of the accretion disk. The two black holes are not resolved in current observations; the required resolution is $\sim 10 \mu$ arcsec. However, the model explains all observations from radio to X-rays and the time variability of these data. 


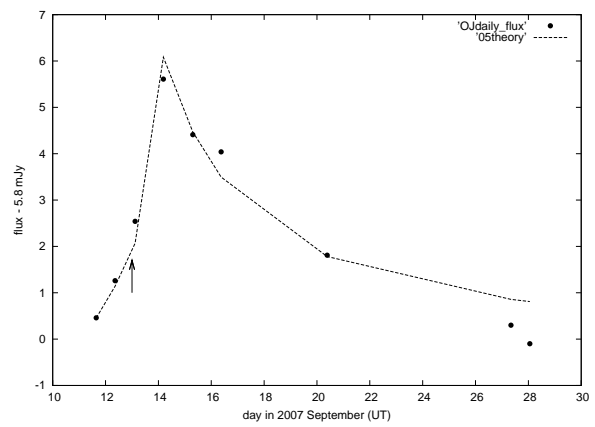

Figure 7. The optical light curve of OJ287 during the 2007 September outburst. Only low polarization (less than 10\%) data points are shown. The dashed line is the theoretical fit. The arrow points to September 13.0, the predicted time of origin of the rapid flux rise.

Lehto and Valtonen (1996) found a solution from five flares which already proved the case in the first approximation.

At a deeper level one has to consider the astrophysical processes that generate the flares. The flares start very suddenly, with the rise time of only about one day (Figure 7). This fact alone excludes many possibilities that one might be otherwise tempted to consider: Doppler boosting variation from a turning jet or increased accretion rate due to varying tidal force. The timescales associated with these processes are months to years quite independent of a detailed model. It turns out that the base level of emission of OJ287, which is synchrotron radiation from the jet, is affected by both of these mechanisms. The Doppler boosting variation accounts for the 55 year cycle (Valtonen and Pihajoki 2013) while the varying tides change the base level in a 11.8 year cycle (Sundelius et al. 1997). Also the $\sim 50$ day cyclic component is due to tides (Figure 8 ), but via a density wave at the innermost stable orbit of the primary accretion disk (Pihajoki et al. 2013).

Figure 9 illustrates the light curve "hump" arising from increased accretion during the 2007 close approach of the two black holes. This feature is well modeled by simulations, and its time scale is about one year. In the same timescale the impact flare is a sharp "spike" on top the hump.

The correct astrophysical explanation of the flares is that the impact on the accretion disk releases hot bubbles of gas from the disk (Ivanov et al. 1998). These bubbles expand until they become optically thin, and the radiation from the whole volume is seen. The calculated light curve profiles agree with this model (Figure 7). The radiation is thermal bremsstrahlung at the temperature of about $3 \times 10^{5} \mathrm{~K}$ (Valtonen et al. 2012). The addition of an unpolarised component to the emission lowers the degree of polarization (Valtonen et al. 2008b) which is another piece of evidence pointing to bremsstrahlung radiation. In contrast, the radiation from the "hump" is clearly synchrothron radiation with a raised number density of radiating particles (Seta 


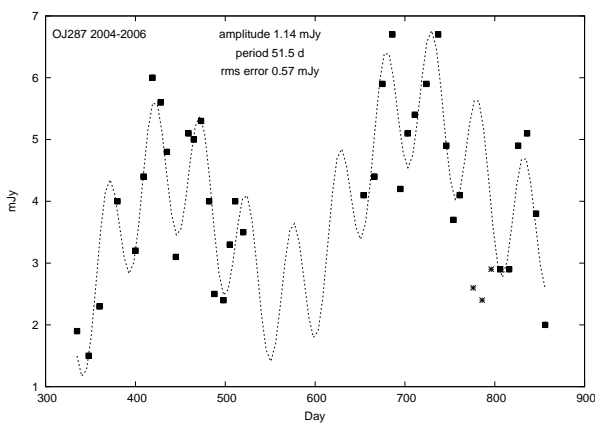

Figure 8. The optical light curve of OJ287 during 2004-2006, using 10 day averages (squares). The 2005 impact flare is excluded. The three stars are points which are not used in the periodicity fit. The best fit for the remaing data is a 51.5 day period. If interpreted as the half-period of the innermost stable orbit, this implies $\chi_{1}=0.25$.

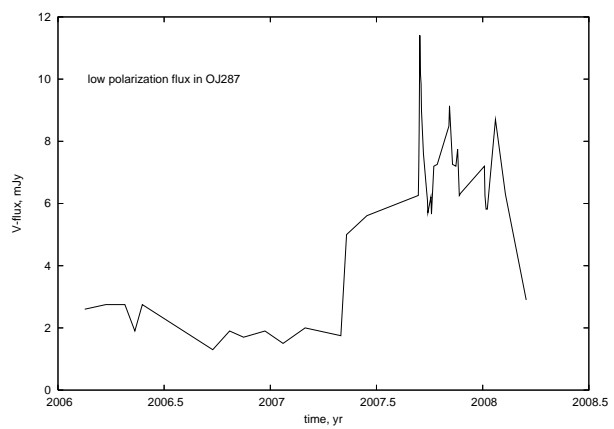

Figure 9. The optical light curve of OJ287 during 2006-2008. Only low polarization (less than $10 \%$ ) data points are shown. 
et al. 2009). OJ287 is the only quasar which is known to have bremsstrahlung flares, and as such these flares give a unique set of signals to be used in orbit determination.

What is more important, the model is able to predict future outbursts. The prediction for the latest outburst was 2007 September 13 (Valtonen 2007, 2008a), accurate to one day, leaving little doubt about the capability of the model (see Figure 7; the predicted time refers in this case to the start of the rapid flux rise.) The 2007 September 13 outburst was an observational challenge, as the source was visible only for a short period of time in the morning sky just before the sunrise. Therefore a coordinated effort was made starting with observations in Japan, then moving to China, and finally to central and western Europe. The campaign was a success and finally proved the case for the binary model (Valtonen et al. 2008b).

We see from Figure 7 that the observed flux rise coincides within 6 hours with the expected time. The accuracy is about the same with which we were able to predict the return of Halley's comet in 1986 !

The astrophysical model introduces a new unknown parameter, the thickness of the accretion disk. For a given accretion rate which is determined from the brightness of the quasar (considering the likely Doppler boosting factor), the thickness is a function of viscosity in the standard $\alpha$ disk theory of Shakura and Sunyaev (1973) and in its extension to magnetic disks by Sakimoto and Corotini (1981). The value of the viscosity coefficient $\alpha \sim 0.3$ is rather typical for other accreting systems (King et al. 2007). Different values of $\alpha$ lead to different delay times between the disk impact and the optical flare. The delay can be calculated exactly except for a constant factor; this factor is an extra parameter in the model. The problem remains mathematically well defined. In fact, using only 6 outbursts as fixed points in the orbit, it is possible to solve the four orbital parameters plus the time delay parameter (in effect $\alpha$ ) in the first approximation (Valtonen 2007). The success of this model in predicting the 2007 outburst was encouraging.

The future optical light curve of OJ287 was predicted from 1996 to 2030 by Sundelius et al. (1997); they published the expected optical flux of OJ287 for every two-week interval between 1900 and 2030. During the first fifteen years OJ287 has followed the prediction with amazing accuracy, producing five outbursts at expected times, of expected light curve profile and size. It is extremely unlikely that such a coincidence should have happened by chance: these optical flux variations alone have excluded alternative models such as quasiperiodic oscillations in an accretion disk at the $5 \sigma$ confidence level (Valtonen et al. 2011b).

OJ287 is unresolved in optical, but in radio and X-ray wavelengths we observe a long jet. The radio jet has been observed since early 1980's and its orientation shows interesting, but in no way simple 12 year cycles. In Figure 10 we show the position angle (PA) observations of the jet as a function of time (Valtonen and Wiik 2012). The line follows a model where the binary shakes the accretion disk gravitationally. This shaking is transmitted to the jet. With suitably chosen parameters the binary action explains not only the cm-wave observations of Figure 10, but also quite different mm- 


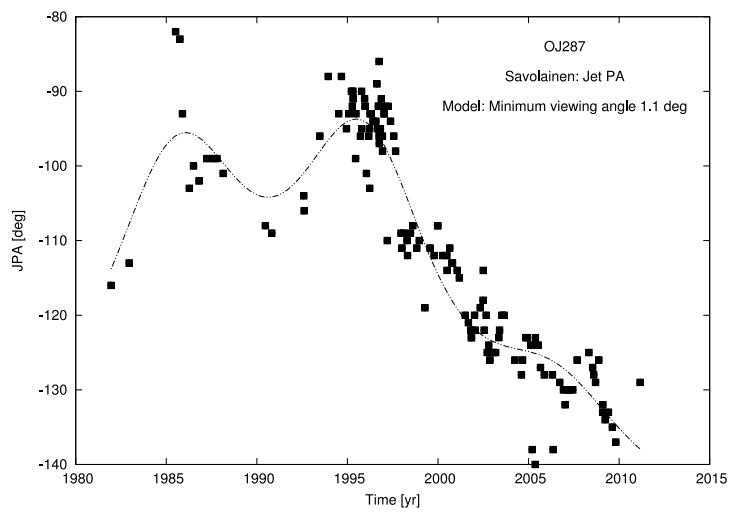

Figure 10. Observations of the jet position angle in OJ287 at $\mathrm{cm}$ wavelengths. The line represents a model where the orientation change propagates outward in the jet with speed $0.85 c$. In observer's time, it takes over 200 years to propagate the change from the optical core to the cm-wave jet. Due to the small viewing angle of $\sim 2^{\circ}$ of the jet, the small changes in its direction are magnified. 
wave jet wobble, in addition to the changes in the optical polarization angle (Valtonen and Pihajoki 2013). All these phenomena occur in the jet, far from the primary sites of action, and thus they cannot be used for high precision determination of the orbit.

The orbital parameters may be determined from the basic binary model without further knowledge of the orbit solution. The precession rate may be estimated by taking the ratio of the two dominant variability frequencies in the optical light curve, averaged over one month to minimize the effects of the impact flares on the analysis. If one of the frequencies $(11.8 \mathrm{yr}$ ) relates to the orbital period while the other frequency (55 yr) arises from precession (Valtonen et al. 2006), then their ratio will tell what fraction of the angle $\pi$ the major axis of the orbit precesses per period. Due to the symmetry of the accretion disk relative to its midplane, the precessional effects should repeat themselves after rotation by $180^{\circ}$. Therefore the precession rate per orbital period should be $11.8 \times 180 / 55=38{ }^{\circ} 6$. Once the precession rate is known, we get a good first estimate for the mass of the primary $m_{1} \sim 1.8 \cdot 10^{10} M_{\odot}$.

The secondary mass was determined by Lehto and Valtonen (1996), $m_{2}=1.44$. $10^{8} M_{\odot}$, when adjusted to the 'current' Hubble constant of $70 \mathrm{~km} / \mathrm{s} / \mathrm{Mpc}$ and to the $5.6 \mathrm{mJy}$ outburst strength, as observed in 2007. The mass value is based on the astrophysics of the impact and the strength of the maximum signal (Ivanov et al. 1998, Valtonen et al. 2012). A large mass ratio is necessary for the stability of the accretion disk. The primary black hole needs to be at least $\sim 130$ times more massive than the secondary (Valtonen et al. 2012). The values of $m_{1}$ and $m_{2}$ satisfy this requirement.

The moderately high eccentricity $e \sim 0.7$ is expected at a certain stage of inspiral of binary black holes of large mass ratio (Baumgardt et al. 2006, Matsubayashi et al. 2007, Iwasawa et al. 2011). Dynamical interaction with the stars of the galactic nucleus drives the eccentricity to $e \sim 0.99$ before the gravitational radiation takes over in the evolution of the binary major axis. When the binary is at the evolutionary stage where $\mathrm{OJ} 287$ is now, the eccentricity has dropped to $e \sim 0.7$.

The value of $\chi_{1}$ may be determined from the period of the innermost stable orbit. The best determination for this period is $103 \pm 4$ days which corresponds to $\chi_{1}=$ $0.25 \pm 0.04$.

The first two columns of Table 5 summarize the parameters determined by astrophysics of the binary black hole system. No information on the outburst timing has been used in the astrophysical model.

\section{Modeling with Post Newtonian methods}

The Post Newtonian approximation to General Relativity provides the equations of motion of a compact binary as corrections to the Newtonian equations of motion in powers of $(v / c)^{2} \sim G M /\left(c^{2} r\right)$, where $v, M$, and $r$ are the characteristic orbital velocity, the total mass, and the typical orbital separation of the binary, respectively. The approximation may be extended to different orders. The terminology 2PN, for example, refers to corrections to Newtonian dynamics in powers of $(v / c)^{4}$. Valtonen et al. 
(2010a) use the 2.5PN and Valtonen et al. (2011a) 3PN-accurate orbital dynamics that includes the leading order general relativistic, classical spin-orbit and radiation reaction effects for describing the evolution of a binary black hole (Kidder 1995). The following differential equations describe the relative acceleration of the binary and the precessional motion for the spin of the primary black hole at $2.5 \mathrm{PN}$ order:

$$
\begin{aligned}
& \ddot{\mathbf{x}} \equiv \frac{d^{2} \mathbf{x}}{d t^{2}}=\ddot{\mathbf{x}}_{0}+\ddot{\mathbf{x}}_{1 P N}+\ddot{\mathbf{x}}_{S O}+\ddot{\mathbf{x}}_{Q} \\
& +\ddot{\mathbf{x}}_{2 P N}+\ddot{\mathbf{x}}_{2.5 P N} \text {, } \\
& \frac{d \mathbf{s}_{1}}{d t}=\boldsymbol{\Omega}_{S O} \times \mathbf{s}_{1},
\end{aligned}
$$

where $\mathbf{x}=\mathbf{x}_{1}-\mathbf{x}_{2}$ stands for the center-of-mass relative separation vector between the black holes with masses $m_{1}$ and $m_{2}$ and $\ddot{\mathbf{x}}_{0}$ represents the Newtonian acceleration given by

$$
\ddot{\mathbf{x}}_{0}=-\frac{G m}{r^{3}} \mathbf{x},
$$

$m=m_{1}+m_{2}$ and $r=|\mathbf{x}|$. Kerr parameter $\chi_{1}$ and the unit vector $\mathbf{s}_{1}$ define the spin of the primary black hole by the relation $\mathbf{S}_{1}=G m_{1}^{2} \chi_{1} \mathbf{s}_{1} / c$ and $\chi_{1}$ is allowed to take values between 0 and 1 in general relativity. $\Omega_{S O}$ provides the spin precessional frequency due to spin-orbit coupling. The PN contributions occurring at the conservative $1 \mathrm{PN}, 2 \mathrm{PN}$ and the reactive $2.5 \mathrm{PN}$ orders, denoted by $\ddot{\mathbf{x}}_{1 P N}, \ddot{\mathbf{x}}_{2 P N}$ and $\ddot{\mathbf{x}}_{2.5 P N}$, respectively, are non-spin by nature. The explicit expressions for these contributions, suitable for describing the binary black hole dynamics, are (Mora and Will 2004)

$$
\begin{array}{cc}
\ddot{\mathbf{x}}_{1 P N}= & -\frac{G m}{c^{2} r^{2}}\left\{\hat { \mathbf { n } } \left[-2(2+\eta) \frac{G m}{r}\right.\right. \\
+ & \left.\left.+3 \eta) v^{2}-\frac{3}{2} \eta^{2}\right]-2(2-\eta) \dot{r} \mathbf{v}\right\}, \\
\ddot{\mathbf{x}}_{2 P N}=\quad-\frac{G m}{c^{4} r^{2}}\left\{\hat { \mathbf { n } } \left[\frac{3}{4}(12+29 \eta)\left(\frac{G m}{r}\right)^{2}\right.\right. \\
+\eta(3-4 \eta) v^{4}+\frac{15}{8} \eta(1-3 \eta) \dot{r}^{4} \\
-\frac{3}{2} \eta(3-4 \eta) v^{2} \dot{r}^{2}-\frac{1}{2} \eta(13-4 \eta)\left(\frac{G m}{r}\right) v^{2} \\
\left.-\left(2+25 \eta+2 \eta^{2}\right)\left(\frac{G m}{r}\right) \dot{r}^{2}\right] \\
-\frac{1}{2} \dot{r} \mathbf{v}\left[\eta(15+4 \eta) v^{2}-\left(4+41 \eta+8 \eta^{2}\right)\left(\frac{G m}{r}\right)\right. \\
\left.\left.-3 \eta(3+2 \eta) \dot{r}^{2}\right]\right\},
\end{array}
$$




$$
\begin{gathered}
\ddot{\mathbf{x}}_{2.5 P N=} \frac{8}{15} \frac{G^{2} m^{2} \eta}{c^{5} r^{3}}\left\{\left[9 v^{2}+17 \frac{G m}{r}\right] \dot{r} \hat{\mathbf{n}}\right. \\
\left.-\left[3 v^{2}+9 \frac{G m}{r}\right] \mathbf{v}\right\},
\end{gathered}
$$

where the vectors $\hat{\mathbf{n}}$ and $\mathbf{v}$ are defined to be $\hat{\mathbf{n}} \equiv \mathbf{x} / r$ and $\mathbf{v} \equiv d \mathbf{x} / d t$, respectively, while $\dot{r} \equiv d r / d t=\hat{\mathbf{n}} \cdot \mathbf{v}, v \equiv|\mathbf{v}|$ and the symmetric mass ratio $\eta=m_{1} m_{2} / \mathrm{m}^{2}$.

The leading order spin-orbit contributions to $\ddot{\mathrm{x}}$, appearing at 1.5PN order (Barker and O'Connell 1975a), read

$$
\begin{aligned}
\ddot{\mathbf{x}}_{S O} & =\frac{G m}{r^{2}}\left(\frac{G m}{c^{3} r}\right)\left(\frac{1+\sqrt{1-4 \eta}}{4}\right) \\
& \chi_{1}\left\{\left[12\left[\mathbf{s}_{1} \cdot(\hat{\mathbf{n}} \times \mathbf{v})\right]\right] \hat{\mathbf{n}}\right. \\
+ & {[(9+3 \sqrt{1-4 \eta}) \dot{r}]\left(\hat{\mathbf{n}} \times \mathbf{s}_{1}\right) } \\
& \left.-[7+\sqrt{1-4 \eta}]\left(\mathbf{v} \times \mathbf{s}_{1}\right)\right\},
\end{aligned}
$$

while

$$
\boldsymbol{\Omega}_{S O}=\left(\frac{G m \eta}{2 c^{2} r^{2}}\right)\left(\frac{7+\sqrt{1-4 \eta}}{1+\sqrt{1-4 \eta}}\right)(\hat{\mathbf{n}} \times \hat{\mathbf{v}}) .
$$

Finally, the quadrupole-monopole interaction term $\ddot{\mathbf{x}}_{Q}$, entering at the $2 \mathrm{PN}$ order (Barker and O'Connell 1975a), reads

$$
\begin{gathered}
\ddot{\mathbf{x}}_{Q}=-q \chi_{1}^{2} \frac{3 G^{3} m_{1}^{2} m}{2 c^{4} r^{4}}\left\{\left[5\left(\hat{\mathbf{n}} \cdot \mathbf{s}_{\mathbf{1}}\right)^{2}-1\right] \hat{\mathbf{n}}\right. \\
\left.-2\left(\hat{\mathbf{n}} \cdot \mathbf{s}_{\mathbf{1}}\right) \mathbf{s}_{\mathbf{1}}\right\},
\end{gathered}
$$

where the parameter $q$, whose value is 1 in general relativity, is introduced to test the black hole 'no-hair' theorems (Will 2008).

It turns out that adding the $3 \mathrm{PN}$ contributions to $d^{2} \mathbf{x} / d t^{2}$, are not necessary; their influence falls within error limits of the OJ287 problem. It has been important to verify that the PN series converges rapidly enough to ingore the terms of order higher than 2.5PN. Similarly, the terms related to the spin of the secondary turn out be negligle in 


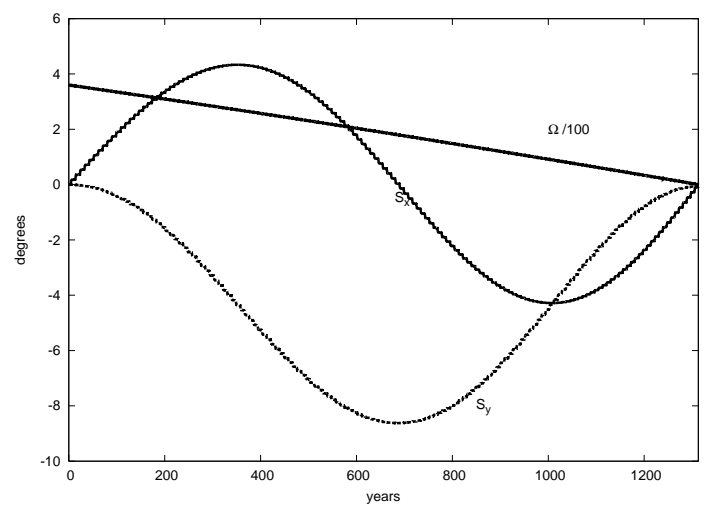

Figure 11. The circulation of the ascending node $(\Omega)$ of the orbit (straight line) in the coordinate system in which the initial spin vector $\mathbf{s}_{\mathbf{1}}$ points to the direction of the z-axis. The quantities plotted are $\Omega / 100$ in degrees and the symbols $s_{x}$ and $s_{y}$ correspond to the $x$ and $y$ components of the spin-direction vector $\mathbf{s}_{\mathbf{1}}$ in degrees.

the OJ287 problem (Valtonen et al. 2011a). The precessional motion for the spin of the primary black hole is $2 \mathrm{PN}$ accurate in our calculations.

The main consequence of including the leading order spin-orbit interactions to the dynamics of a binary black hole is that it forces the orbital plane to precess. The orbital angular momentum vector, characterising the orbital plane, precesses around the spin of the primary in such a way that the angle between the orbital plane and the spin vector $\mathbf{s}_{1}$ remains almost constant (roughly within \pm 0.5 in the model). The spin-vector itself precesses drawing a cone with an opening angle of about 8 degrees (see Figure 11).

The precessional period for both the orbital plane and the spin of the binary, provided by $\left|\boldsymbol{\Omega}_{S O}\right|$, is about 1300 years for the orbital parameters of OJ287. The precession cone axis coincides with the mean accretion disk axis. It is reasonable to expect such a situation due to the Bardeen-Petterson effect (Kumar and Pringle 1985). Because the time scale of the Bardeen-Petterson effect is much longer than the black hole spin precession time scale (Lodato and Pringle 2006), the disk axis is not able to follow the rapid precession of the primary spin axis.

The orbit solutions currently involve nine accurately timed outbursts as listed in Table 6. The orbit solutions provide a unique set of parameters (Table 5, column 3). Recently also the tenth outburst from 1906 has been added (Hudec et al. 2013). In this case there are only limits for the timing; the main influence of including the 1906 data is to bring $\chi_{1}$ to $\sim 0.23$, somewhat below the range given in Table 5. Note that the eccenticity $e$ is defined as

$$
\frac{r_{a}}{r_{p}}=\frac{1+e}{1-e},
$$

where $r_{a}$ and $r_{p}$ are the apocenter and pericenter distances in the orbit, respectively. 
The instantaneous eccentricity varies during a relativistic binary orbit; in this way we get a definite number that resembles the eccentricity of a Keplerian orbit.

Because we are using 8 time intervals to fit 8 parameters, the fit has no degrees of freedom. The tolerance limits of Table 5 are a consequence of having a tolerance in the fixed points of the outburst times (Table 6). The no-hair parameter $q$ is the eigth parameter which of course should be $q=1$ if General Relativity is correct.

As part of the solution we obtain the list of all past and future outburst times with their uncertainties. For example, the well recognized outbursts in 1959, 1971 and 1994 are timed at $1959.213 \pm 0.002,1971.1265 \pm 0.002$ and $1994.6085 \pm 0.005$, respectively. In all these cases data are missing at the crucial time of expected rapid flux rise, and thus these predictions cannot be verified at present.

It is remarkable that the two sets of parameters in second and third columns of Table 1 agree so closely. If the basic model were not correct, there would be no reason for this agreement. Also, the mere fact that an orbit solution exists at all is a strong argument for the model.

Let us now turn our attention to the expected future outbursts in OJ287. We expect three more outbursts during the next two decades, occuring in 2016, 2019 and 2022. The 2016 outburst should be an easy one to detect, as it comes in January of that year. Its timing is spin-sensitive; the exact date will give us a good spin value from the following formula:

$$
\chi_{1}=0.25-0.5 \times(t-2016.0) .
$$

Here $t$ is the time of the beginning of the outburst in years. The expected accuracy is 0.005 units in $\chi_{1}$. The dependence on $q$ is secondary, and thus the 2016 outburst timing is of no use by itself for the testing of the no-hair theorem.

The value of $q$ is currently best determined by the 1995 outburst. There was an intensive monitoring campaign of OJ287 (called OJ94) during this outburst season, but unfortunately there exists a gap in these observations just at the crucial time (Valtonen 1996, Figure 12). It may still be possible that there are measurements somewhere which are not recorded in the OJ94 campaign light curve, and which would be valuable in narrowing down $q$ even from these data. The line in Figure 12 is drawn using the well observed 2007 outburst as a template to compare with 1995. It is should be noted that even a few more measurements of 1995 could narrow down the range of $q$.

The 2019 outburst timing is also sensitive to the $q$ :

$$
q=1.0-1.2 \times\left(t-t_{0}\right),
$$

where $t$ is time of the beginning of the outburst (in days) and $t_{0}$ is 2019 July 14, 11:19 UT. The expected accuracy for the $q$ determination is 0.15 units, i.e. optimally the accuracy of the no-hair theorem test is doubled in comparison with the present uncertainty. Observing OJ287 during the rising flux of the 2019 outburst, from July 14 to July 17, is a challenge for ground based observers. This is because the angular 


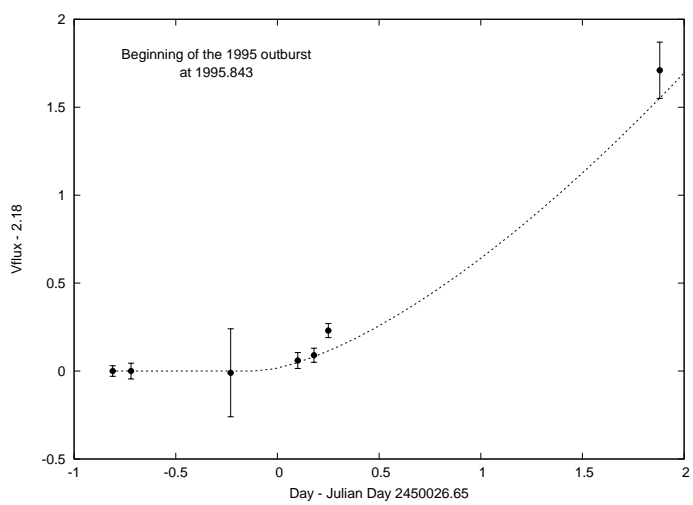

Figure 12. The observations of OJ287 during 4 - 7 November 1995, transformed to optical V-band. Overlaid is the theoretical light curve profile, corresponding to $q=1$. The zero point of the time axis is at 3:36 UT of 1995, November 5. The peak flux of 5.1 mJy above the base level was predicted for November 8 (Lehto and Valtonen 1996), but there were no observations to confirm it. The data on November 9 and later suggest that the outburst maximum was probably missed.

distance between the Sun and OJ287 is only about 19 degrees at the beginning of the event, and goes down to about 16 degrees by the time of the peak flux on July 17. T. Pursimo has measured OJ287 at the Nordic Optical telescope on 2007 July 12 (Valtonen et al. 2009) which demonstrates that the July measurements should also be feasible in 2019. Space observations would also be useful in order to study the outburst over a wider spectral range. The 2022 outburst is scheduled practically at the same time of year as the 2019 outburst. Obviously it would also be of interest to observe this event as it would tie down the parameters of the general model more narrowly. However, it will not give any further information on $q$.

\section{$90 \mathrm{~J} 287$ results at the strong field limit}

The binary black hole in OJ287 is modeled to contain a spinning primary black hole with an accretion disk and a spinning or non-spinning secondary black hole. Using $\mathrm{PN}$-accurate dynamics, relevant for such a system, we infer that the primary black hole should spin approximately at one quarter of the maximum spin rate allowed in general relativity. In addition, the 'no-hair theorems' of black holes (Misner et al. 1973) are supported by the model, although the testing is possible only to a limited precision. The solutions concentrate around $q=1.0$ with one standard deviation of 0.3 units. These results are achieved with the help of new data on historical outbursts as well as using the most recent outburst light curves together with the timing model for OJ287 outbursts (Valtonen et al. 2007).

Further, a polar orbit is assumed. Any other high inclination model would give us 
the same timing results. Sundelius et al. (1997) carried out simulations with a number of different inclinations between the disk and the orbit, and found that the inclination makes no difference. This is not surprising since the impacts occur along the line of nodes, and the timing of the impacts is not a function of the impact angle. We have tried varying the spin angle relative to the disk, and found that within reasonable limits (we considered inclinations up to $10^{\circ}$ ) the results do not change. Again this is what is expected since the line of nodes circulates slowly in comparison with the orbital angular motion.

The reason why we would expect at least approximate alignment between the black hole spin and the disk spin is the Bardeen - Petterson effect which tends to align the two spins. However, the alignment time scale is much slower (about $10^{7} \mathrm{yr}$, Lodato \& Pringle 2006) than the spin precession time, and thus the disk does not follow the black hole spin exactly but only on average.

In this scenario, we have a unique solution and also a unique prediction for the next OJ287 outburst, expected in 2016. We should then be able to judge the correctness of the present solution. Note that an outburst is not expected in 2016 in any simple extrapolation from past observations, as it is well before the average $12 \mathrm{yr}$ cycle is due, and thus it is a sensitive test of the general model as well as a test for the spin of the primary black hole. The observations of the 2019 outburst will then improve the accuracy of the 'no-hair' theorem test by a factor of two, to the level of $15 \%$.

There are at least 5 additional outbursts in the historical record which have not yet been been accurately observed. If new data of these outbursts are found it will open up the possibility of improving the model and improving that accuracy of the no-hair theorem test to the level of $10 \%$. Searches of plate archives are recommended for this purpose.

\section{Summary and Conclusions on General Relativity at weak and strong field limits}

We have discussed the amazing range of validity of General Relativity from tests from the weak to strong field limits. The weak field corresponds to speeds much less than that of light or distances much larger than the Schwarzschild radius of the primary body of an orbiting pair. In weak field tests, we briefly reviewed the precession of Mercury's orbit, bending of light near the Sun, precession of a binary pulsar orbit, gravitational radiation from the binary pulsar, relativistic geodetic precession and the precession due to the relativistic Lense-Thirring effect. In all these cases General Relativity accounts for the observations but, the cosmological constant (manifested as dark energy) is not significant.

However, the existence of dark energy in General Relativity can be tested in the weak field limit. Einstein (1917) proposed a cosmological constant specified to produce a static universe. Evolving models of the universe were derived by Alexander 
Friedmann (1922, 1924) and Georges Lemaitre (1927). The Einstein static solution is a special case, where the cosmological constant is related to the matter density to give gravity-antigravity balance. In general there are no theoretical restrictions on the cosmological constant. The universe expands or contracts as a whole. The Hubble discovery of the redshift distance relation (Hubble 1929) can be regarded as a predictive verification of General Relativity.

After the discovery of the Big Bang, it seemed that the cosmological constant could not be as big as Einstein required for a static universe. It was subsequently commonly assumed to be zero. However, when supernovae of type Ia were used to estimate distances of galaxies whose redshifts $z \sim 1$, a positive cosmological constant was indicated (Riess et al. 1998 and Perlmutter et al. 1999).

We describe in detail how to test the existence of dark energy at the weak field limit of General Relativity via an outflow model in groups and clusters of galaxies (Chernin 2001; Chernin et al. 2006; Byrd et al. 2011). The Local Group has been found to have outflowing dwarf galaxies around it (van den Bergh 1999; Karachentsev et al. 2009). The dwarfs' motion indicates not only the gravitational mass of the group but also the dark energy background (Chernin et al. 2009). Studies the Virgo and Fornax clusters and even rich clusters like Coma (Karachentsev et al. 2003, Chernin 2008, Chernin et al. 2006, 2007, 2010, 2012a,b, Chernin 2013) show similar structure.

There has been been a large amount of effort to determine the value of the cosmological constant, and its possible dependence on redshift or, equivalently, time (Frieman et al. 2008, Blanchard 2010, Weinberg et al. 2013). In the literature from year 2005 onwards, values cluster around $\Omega_{\Lambda}=0.73$ with a standard deviation of 0.044 , the typical error in individual measurements. Only the cosmic microwave background (CMB) models give a significantly better accuracy, but even in this case one may suspect hidden systematic errors due to foreground corrections (Whitbourn et al. 2014). In our analysis of the literature including nearby groups and clusters, within the error limits, $\Lambda$ appears to be a constant over the redshift range from cosmological to Local Group outflows, an impressive validation.

The strong field corresponds to speeds comparable to that of light or distances comparable to the Schwarzschild radius of the primary body of a pair. An important strong field test is to be sure that black holes are indeed the singularities predicted in General Relativity. We have to ascertain that at least in one case the spacetime around a suspected black hole satisfies the no-hair theorems which state that an electrically neutral rotating black hole in General Relativity is completely described by its mass, $M$, and its angular momentum, $S$, implying that the multipole moments, required to specify the external metric are fully expressible in terms of $M$ and $S$. No-hair theorems apply only in General Relativity, and thus are a powerful eliminator of various alternative theories of gravitation (Will 2006, Yunes and Siemens 2013, Gair et al. 2013).

An ideal test of the no-hair theorem is to have a test particle in orbit around a spinning black hole. We have described results for one such system, the BL Lacertae object OJ287, a binary black hole of very large mass ratio. OJ287 gives outbursts during the 
secondary's periodic orbital disk crossings of an accretion disk around the more massive member. The timing of the repeating outbursts gives the orbit and the primary's quadrupole parameter. Post-Newtonian dynamics, necessary for such a system, implies that the primary black hole should spin at about one quarter of the maximum spin rate allowed in General Relativity. The 'no-hair theorems' of black holes (Misner et al. 1973) are supported by the model but with a limited precision at present (Valtonen et al. 2011a). Observations of predicted 2016 and 2019 outbursts and collection of data on more past outbursts should improve the accuracy of the 'no-hair' theorem test. This extreme test at the strong field limit supports General Relativity.

Acknowledgments. We note that Bruce Barker (who died in 1988 at the age of 54) was a co-author of two important theoretical papers testing General Relativity in extreme binary systems like those discussed here. He was a friend and colleague of $\mathrm{M}$. V. and G. B. at the University of Alabama.

\section{Bibliography}

[1] A. Einstein, Die Grundlage der allgemeinen Relativitätstheorie, Annalen der Physik, 354 (1916), 769-822.

[2] A. Einstein, Kosmologische Betrachtungen zur allgemeinen Relativitätstheorie, Sitzungsberichte der Königlich Preussischen Akademie der Wissenschaften (Berlin), (1917), 142152.

[3] G.G. Byrd, A.D. Chernin and M.J. Valtonen, Cosmology: Foundations and Frontiers, Moscow, URSS (2007).

[4] G. Byrd, A. D. Chernin, P. Teerikorpi and M. Valtonen, Paths to Dark Energy, De Gruyter, (2012).

[5] G. Lemaittre, The expanding universe, Monthly Notices of the Royal Astronomical Society, 91 (1931), 490-501.

[6] G.C. McVittie, Condensations in an expanding universe, Monthly Notices of the Royal Astronomical Society, 92 (1932), 500-518.

[7] G.C. McVittie, The mass-particle in an expanding universe, Monthly Notices of the Royal Astronomical Society, 93 (1933), 325-339.

[8] G. Järnefelt, Über die Bewegung eines Massenpunktes in einem Raum mit zentralsymmetrischer Massen- und Druckverteilung, Zeitschrift für Astrophysik, 7 (1933), 326-327.

[9] A. Einstein and E. G. Straus, The Influence of the Expansion of Space on the Gravitation Fields Surrounding the Individual Stars, Reviews of Modern Physics, 17 (1945), 120-124.

[10] A. Einstein and E.G. Straus, Corrections and Additional Remarks to our Paper: The Influence of the Expansion of Space on the Gravitation Fields Surrounding the Individual Stars, Reviews of Modern Physics, 18 (1946), 148-149.

[11] D. Psaltis, Probes and Tests of Strong-Field Gravity with Observations in the Electromagnetic Spectrum, Living Reviews in Relativity, 11 (2008), 9:1-9:61. 
[12] U. Le Verrier, Lettre de M. Le Verrier à M. Faye sur la theorie de Mercure et sur le mouvement du perihelie de cette planete Comptes rendus hebdomadaires des seances de l'Academie des sciences (Paris), 49 (1859), 379-383.

[13] S. Newcomb, The elements of the four inner planets and the fundamental constants, (Washington, D. C., U. S. Govt. Office, 1895).

[14] A. Hall, A suggestion in the theory of Mercury, Astron. J., 14 (1894), 49-51.

[15] I. I. Shapiro, C. C. Counselman III and R. W. King, Verification of the principle of equivalence for massive bodies, Phys. Rev. Lett., 36 (1976), 555-558.

[16] A. Einstein, Erklärung der Perihelbewegung des Merkur aus der allgemeinen Relativitätstheorie Sitz. Preuss. Akad. Wiss., 11 (1915), 831-839.

[17] R. A. Hulse and J. H. Taylor, Discovery of a pulsar in a binary system, Astrophys. J., 195 (1975), L51-L53.

[18] J. H. Taylor and J. M. Weisberg, Further experimental tests of relativistic gravity using the binary pulsar PSR $1913+16$, Astrophys. J., 345 (1989), 434-450.

[19] T. Damour and J. H. Taylor, On the orbital period change of the binary pulsar PSR1913+16, Astrophys. J., 366 (1991), 501-511.

[20] R. A. Hulse, The discovery of the binary pulsar, Rev. Mod. Phys., 66 (1994), 699-710.

[21] A. G. Lyne et al., A Double-Pulsar System: A Rare Laboratory for Relativistic Gravity and Plasma Physics, Science, 303 (2004), 1153-1157.

[22] M. Kramer et al., Tests of General Relativity from Timing the Double Pulsar, Science, 314 (2006), 97-102.

[23] J. Lense and H. Thirring, Über den Einfluss der Eigenrotation der Zentralkörper auf die Bewegung der Planeten und Monde nach der Einsteinschen Gravitationstheorie. Physikalische Zeitschrift, 19 (1918), 156-163.

[24] I. Cuifolini and E. C. Pavlis, A confirmation of the general relativistic prediction of the Lense-Thirring effect, Nature, 431 (2004), 958-960.

[25] C. W. F. Everitt et al., Gravity Probe B: Final Results of a Space Experiment to Test General Relativity, Physical Review Letters, 106 (2011), 221101-1-221101-5

[26] T. Damour and R. Ruffini, Certain new verifications of general relativity made possible by the discovery of a pulsar belonging to a binary system, Acad. Sci. Paris Comptes Rendus Ser. Sci. Math., 279 (1974), 971-973.

[27] B. M. Barker and R. F. O'Connell, Gravitational two-body problem with arbitrary masses, spins, and quadrupole moments, Physical Review D, 12 (1975a), 329-335.

[28] B. M. Barker and R. F. O'Connell, Relativistic effects in the binary pulsar PSR 1913+16, Astrophys.J.Lett., 199 (1975b), L25-L26.

[29] J. M. Weisberg and J. H. Taylor, 2005, The Relativistic Binary Pulsar B1913+16: Thirty Years of Observations and Analysis, Binary Radio Pulsar, ASP Conference Series, Editors: F. A. Rasio and I. H. Stairs, 328 (2005), 25-31. 
[30] J. M. Weisberg, D. J. Nice and J. H. Taylor, 2010, âĂIJTiming Measurements of the Relativistic Binary Pulsar B1913+16 âĂİ Astrophys.J., 722 (2010), 1030-1034.

[31] A. Einstein, Über den Einfluss der Schwerkraft auf die Ausbreitung des Lichtes Annalen der Physik, 340 (1911), 898-908.

[32] A. S. Eddington, The total eclipse of May 29 and the influence of gravitation on light, Observatory, 42 (1919), 119-122.

[33] F. W. Dyson, A. S. Eddington and C. R. Davidson, C., A Determination of the Deflection of Light by the Sun's Gravitational Field, from Observations Made at the Total Eclipse of May 29, 1919, Philosophical Transactions of the Royal Society of London. Series A, Containing Papers of a Mathematical or Physical Character, 220 (1920), 291-333.

[34] D. Walsh, R. F. Carswell and R. J. Weymann, 0957 + 561 A, B âĂŞ twin quasistellar objects or gravitational lens, Nature, 279 (1979), 381-384.

[35] M. J. Valtonen and H. J. Lehto, Outbursts in OJ 287: A New Test for the General Theory of Relativity, Astrophysical Journal Letters, 481 (1997), L5-L7.

[36] Y.-T. Sun, J.-Y. Liu, J.-Z. Liu, Y.-L. Ai, M. Zhou and E.-L. Qiao, A Research on the Gravitational Wave Radiation of OJ 287, Chinese Astronomy and Astrophysics, Volume 35 (2011), 123-132.

[37] J. Liu, Y. Zhang, H. Zhang, Y. Sun and N. Wang, Determining gravitational wave radiation from close galaxy pairs using a binary population synthesis approach, Astronomy and Astrophysics, Volume 540 (2012), A67:1-A67:6.

[38] R. P. Kerr, Gravitational Field of a Spinning Mass as an Example of Algebraically Special Metrics, Physical Review Letters, 11 (1963), 237-238.

[39] M. J. Valtonen et al., Measuring black hole spin in OJ287, Celestial Mech. and Dynamical Astr., 106 (2010b), 235-243.

[40] W. Israel, Event horizons in static vacuum spacetimes, Phys.Rev. 164 (1967), 1776-1779.

[41] W. Israel, Event horizons in static electrovac spacetimes, Commun. Math.Phys. 8 (1968), 245-260.

[42] B. Carter, An axisymmetric black hole has only two degrees of freedom, Phys.Rev.Lett. 26 (1970), 331-333.

[43] S. W. Hawking, Gravitational radiation from colliding black holes, Phys.Rev.Lett. 26 (1971), 1344-1346.

[44] S. W. Hawking, Black holes in general relativity, Commun.Math.Phys. 25 (1972), 152166.

[45] C. W. Misner, K. S. Thorne and J. A. Wheeler, Gravitation (W.H.Freeman \& Sons, San Francisco, p. 876, 1973).

[46] A. A. Friedmann, Über die Krümmung des Raumes, Zeitschrift für Physik, 10 (1922), 377-386.

[47] A. A. Friedmann, Über die Möglichkeit einer Welt mit konstanter negativer Krümmung des Raumes, Zeitschrift für Physik, 21, 326-332. 
[48] G. Lemaitre, Un Univers homogene de masse constante et de rayon croissant rendant compte de la vitesse radiale des nebuleuses extra-galactiques, Annales de la Societe Scientifique de Bruxelles, A47 (1927), 49-59.

[49] E. Hubble, A Relation between Distance and Radial Velocity among Extra-Galactic Nebulae, Proceedings of the National Academy of Sciences of the United States of America, 15 (1929), 168-173.

[50] A. G. Riess et al., Observational evidence from supernovae for an accelerating universe and a cosmological constant, Astron. J., 116 (1998) 1009-1038.

[51] S. Perlmutter et al., Measurements of Omega and Lambda from 42 High-Redshift Supernovae, Astrophys. J., 517 (1999), 565-586.

[52] A.D. Chernin, Reviews of topical problems: Cosmic vacuum, Physics Uspekhi, 44 (2001), 1099-1118.

[53] A.D. Chernin, P. Teerikorpi and Yu.V. Baryshev, Non-Friedmann cosmology for the Local Universe, significance of the universal Hubble constant, and short-distance indicators of dark energy, Astronomy and Astrophysics, 456 (2006), 13-21.

[54] S. van den Bergh, The local group of galaxies, Astronomy and Astrophysics Review, 9 (1999), 273-318.

[55] I. D. Karachentsev, O. G. Kashibadze, D. I. Makarov and R. B. Tully, The Hubble flow around the Local Group, Monthly Notices of the Royal Astronomical Society, 393 (2009), $1265-1274$.

[56] A.D. Chernin et al., Local dark matter and dark energy as estimated on a scale of $1 \mathrm{Mpc}$ in a self-consistent way, Astronomy and Astrophysics, 507 (2009), 1271-1276.

[57] Yu.V. Baryshev, A.D. Chernin and P. Teerikorpi, The cold local Hubble flow as a signature of dark energy, Astronomy and Astrophysics, 378 (2001), 729-734.

[58] P. Teerikorpi, A.D. Chernin and Yu.V. Baryshev, The quiescent Hubble flow, local dark energy tests, and pairwise velocity dispersion in a $\Omega=1$ universe, Astronomy and Astrophysics, 440 (2005), 791-797.

[59] I.D. Karachentsev et al., Local galaxy flows within $5 \mathrm{Mpc}$, Astronomy and Astrophysics, 398 (2003), 479-491.

[60] P. Teerikorpi, A.D. Chernin, I.D. Karachentsev and M.J. Valtonen, Dark energy in the environments of the Local Group, the M 81 group, and the CenA group: the normalized Hubble diagram, Astronomy and Astrophysics, 483 (2008), 383-387.

[61] A.D. Chernin et al., The very local Hubble flow: Computer simulations of dynamical history, Astronomy and Astrophysics, 415 (2004), 19-25.

[62] S. Peirani and J.A. de Freitas Pacheco, Dynamics of nearby groups of galaxies: the role of the cosmological constant, Astronomy and Astrophysics, 488 (2008), 845-851.

[63] J. Saarinen and P. Teerikorpi, arXiv: 1402.0950 (2013).

[64] M.J. Valtonen, G.G. Byrd, M.L. McCall and K.A. Innanen, A revised history of the Local Group and a generalized method of timing, Astronomical Journal, 105 (1993), 886-893. 
[65] G. Byrd, M. Valtonen, M. McCall and K. Innanen, Orbits of the Magellanic Clouds and Leo I in local group history, Astronomical Journal, 107 (1994), 2055-2059.

[66] A.D. Chernin et al., Local dark energy: HST evidence from the vicinity of the M81/M82 galaxy group, Astrophysics, 50 (2007), 405-415.

[67] P. Teerikorpi and A.D. Chernin, The Hubble diagram for a system within dark energy: the location of the zero-gravity radius and the global Hubble rate, Astronomy and Astrophysics, 516 (2010), A93:1-A93:4.

[68] F.D. Kahn and L. Woltjer, Intergalactic Matter and the Galaxy, Astrophysical Journal, 130 (1959), 705-717.

[69] J. Binney and S. Tremaine, Galactic Dynamics: Second Edition, Princeton University Press, Princeton, NJ USA, (2008).

[70] S. Garrison-Kimmel, M. Boylan-Kolchin, J. Bullock and K. Lee, ELVIS: Exploring the Local Volume in Simulations, arXiv: 1310.6746 (2013).

[71] W.R. Forman, A Reduction of the Mass Deficit in Clusters of Galaxies by Means of a Negative Cosmological Constant, Astrophysical Journal, 159 (1970), p.719-722.

[72] J.C. Jackson, The dynamics of clusters of galaxies in universes with non-zero cosmological constant, and the virial theorem mass discrepancy, Monthly Notices of the Royal Astronomical Society, 148 (1970), 249-260.

[73] A.D. Chernin et al., Virial estimator for dark energy, Gravitation and Cosmology, 18 (2012), 1-5.

[74] M. Valtonen and H. Karttunen, The Three-Body Problem, Cambridge University Press, Cambridge (2006).

[75] A.D. Chernin, Dark energy in the nearby Universe: HST data, nonlinear theory, and computer simulations, Physics-Uspekhi, 56 (2013), 704.

[76] F. Zwicky, Die Rotverschiebung von extragalaktischen Nebeln, Helvetica Physica Acta, 6 (1933), 110-127.

[77] F. Zwicky, On the Masses of Nebulae and of Clusters of Nebulae, Astrophysical Journal, 86 (1937), p.217-246.

[78] L.S. The and S.D.M. White, The mass of the Coma cluster, Astronomical Journal, 92 (1986), 1248-1253.

[79] J.P. Hughes, The mass of the Coma Cluster - Combined X-ray and optical results, Astrophysical Journal, 337 (1989), 21-33.

[80] J.P. Hughes, Untangling Coma Berenices: A New Vision of an Old Cluster, Proceedings of the meeting held in Marseilles, June 17-20, 1997, Eds.: Mazure, A., Casoli F., Durret F. , Gerbal D., Word Scientific, p 175 (1998).

[81] M. Colless, The 2dF Galaxy Redshift Survey, in Encyclopedia of Astronomy and Astrophysics, P.Murdin, ed., Bristol: Institute of Physics Publishing, UK, article 5485 (2002).

[82] J.M. Kubo et al., The Mass of the Coma Cluster from Weak Lensing in the Sloan Digital Sky Survey, The Astrophysical Journal, 671 (2007), 1466-1470. 
[83] M.J. Valtonen and G.G. Byrd, A binary model for the Coma cluster of galaxies, Astrophysical Journal, 230 (1979), 655-666.

[84] M.J. Valtonen, K.A. Innanen, T.-Y. Huang and S. Saarinen, No missing mass in clusters of galaxies?, Astronomy and Astrophysics, 143 (1985), 182-187.

[85] S. Laine, J.-Q. Zheng and M.J. Valtonen, Improved Models for the Evolution of the Coma Cluster of Galaxies, Astronomical Journal, 127 (2004), 765-770.

[86] A. Diaferio and M.J. Geller, Infall Regions of Galaxy Clusters, Astrophysical Journal, 481, 633-643.

[87] A. Diaferio, Mass estimation in the outer regions of galaxy clusters, Monthly Notices of Royal Astronomical Society, 309 (1999), 610-622.

[88] M.J. Geller, A. Diaferio and M.J. Kurtz, The Mass Profile of the Coma Galaxy Cluster, The Astrophysical Journal, 517 (1999), L23-L26.

[89] M.J. Geller, A. Diaferio and M.J. Kurtz, Mapping the Universe: The 2010 Russell Lecture, The Astronomical Journal, 142 (2011), 133:1-133:7.

[90] J.F. Navarro, C.S. Frenk and S.D.M. White, A Universal Density Profile from Hierarchical Clustering, Astrophysical Journal, 490 (1997), 493-508.

[91] L. Hernquist, An analytical model for spherical galaxies and bulges, Astrophysical Journal, 356 (1990), 359-364.

[92] D.E. Holz and S. Perlmutter, The Most Massive Objects in the Universe, The Astrophysical Journal Letters, 755 (2012), L36:1-L36:4.

[93] M.T. Busha, A.E. Evrard, F.C. Adams and R.H. Wechsler, The ultimate halo mass in a $\Lambda \mathrm{CDM}$ universe, Monthly Notices of the Royal Astronomical Society: Letters, Volume $\mathbf{3 6 3}$ (2005), L11-L15.

[94] I.D. Karachentsev, A.D. Chernin and P. Teerikorpi, The Hubble Flow: Why Does the Cosmological Expansion Preserve Its Kinematical Identity from a Few Mpc Distance to the Observation Horizon?, Astrophysics (English translation of Astrofizika), 46 (2003), 399-414.

[95] A.D. Chernin, Physics of our days: Dark energy and universal antigravitation, Physics Uspekhi, 51 (2008), 253-282.

[96] A.D. Chernin, P. Teerikorpi and Yu.V. Baryshev, Why is the Hubble flow so quiet? Advances in Space Research, 31 (2003), 459-467.

[97] A.D. Chernin et al., Dark energy domination in the Virgocentric flow, Astronomy and Astrophysics, $\mathbf{5 2 0}$ (2010), A104:1-A104:7.

[98] A.D. Chernin et al., Dark energy in six nearby galaxy flows: Synthetic phase diagrams and self-similarity, Astronomy Reports, 56 (2012a), 653-663.

[99] A.D. Chernin et al., Dark energy and extended dark matter halos, Astronomy and Astrophysics, 539 (2012b), A4:1-A4:6.

[100] A.D. Chernin, G.S. Bisnovatyi-Kogan, P. Teerikorpi, M.J. Valtonen, G.G. Byrd and M. Merafina, Dark energy and the structure of the Coma cluster of galaxies, Astronomy and Astrophysics, 553 (2013), A101:1-A101:4. 
[101] F.D.A. Hartwick, The Velocity Field around Groups of Galaxies, The Astronomical Journal, 141 (2011), 198:1-198:5.

[102] M. Merafina, G.S. Bisnovatyi-Kogan and S.O. Tarasov, A brief analysis of selfgravitating polytropic models with a non-zero cosmological constant, Astronomy and Astrophysics, 541 (2012), A84:1-A84:5.

[103] G.S. Bisnovatyi-Kogan and A.D. Chernin, Dark energy and key physical parameters of clusters of galaxies, Astrophysics and Space Science, 338 (2012), 337-343.

[104] G.S. Bisnovatyi-Kogan and M. Merafina, Galactic cluster winds in presence of a dark energy, Monthly Notices of the Royal Astronomical Society, 434 (2013), 3628-3632.

[105] S.Saarinen and M.J. Valtonen, Simulations of clusters of galaxies with massive cores, Astronomy and Astrophysics, 153 (1985), 130-138.

[106] G.G. Byrd and M.J. Valtonen, Origin of redshift differentials in galaxy groups, Astrophysical Journal, 289 (1985), 535-539.

[107] M.J. Valtonen and G.G. Byrd, Redshift asymmetries in systems of galaxies and the missing mass, Astrophysical Journal, 303 (1986), 523-534.

[108] S.-M. Niemi and M. Valtonen, The origin of redshift asymmetries: how ÎŻCDM explains anomalous redshift, Astronomy and Astrophysics, 494 (2009), 857-865.

[109] J.A. Frieman, M.S. Turner and D. Huterer, Dark Energy and the Accelerating Universe, Annual Review of Astronomy and Astrophysics, 46 (2008), 385-432.

[110] A. Blanchard, Evidence for the fifth element. Astrophysical status of dark energy, Astronomy and Astrophysics Review, 18 (2010), 595-645.

[111] D.H. Weinberg et al., Observational probes of cosmic acceleration, Physics Reports, 530 (2013), 87-255.

[112] J.R. Whitbourn, T. Shanks and U. Sawangwit, Testing WMAP data via Planck radio and SZ catalogues, Monthly Notices of the Royal Astronomical Society, 437 (2014), 622-640.

[113] A.D. Chernin, Dark Energy in Systems of Galaxies, JETP Letters, 98 (2013), 394-407.

[114] R. Mohayaee and R.B. Tully, The Cosmological Mean Density and Its Local Variations Probed by Peculiar Velocities, Astrophys. J., 635 (2005), L113-L116.

[115] Y.-Z. Ma and D. Scott, Cosmic bulk flows on $50 h^{-1}$ Mpc scales: a Bayesian hyperparameter method and multishell likelihood analysis, Monthly Notices of the Royal Astronomical Society, 428 (2013), 2017-2028.

[116] A. Nusser and M. Davis, The Cosmological Bulk Flow: Consistency with $\Lambda$ CDM and $z \sim 0$ Constraints on $\sigma_{8}$ and $\gamma$, The Astrophysical Journal, 736 (2011), 93:1-93:9.

[117] C.-G. Park and C. Park, Power Spectrum of Cosmic Momentum Field Measured from the SFI Galaxy Sample, Astrophys. J., 637 (2006), 1-11.

[118] M. Kowalski et al., Improved Cosmological Constraints from New, Old, and Combined Supernova Data Sets, Astrophys. J., 686 (2008), 749-778.

[119] J.P. Henry, A.E. Evrard, H. Hoekstra, A. Babul and A. Mahdavi, The X-Ray Cluster Normalization of the Matter Power Spectrum, Astrophysical J., 691 (2009), 1307-1321. 
[120] F. Beutler et al., The $6 \mathrm{dF}$ Galaxy Survey: $z \approx 0$ measurements of the growth rate and $\sigma_{8}$, Monthly Notices of the Royal Astronomical Society, 423 (2012), 3430-3444.

[121] J.L. Tinker et al., The Cosmological Constraints from Galaxy Clustering and the Massto-number Ratio of Galaxy Clusters, Astrophys. J., 745 (2012), 16:1-16:22.

[122] V. Simha and S. Cole, Cosmological constraints from applying SHAM to rescaled cosmological simulations , Monthly Notices of the Royal Astronomical Society, 436 (2013), $1142-1151$.

[123] M. Cacciato, F.C. van den Bosch, S. More, H. Mo and X. Yang, Cosmological constraints from a combination of galaxy clustering and lensing - III. Application to SDSS data, Monthly Notices of the Royal Astronomical Society, 430 (2013), 767-786.

[124] S. Cole et al., The 2dF Galaxy Redshift Survey: power-spectrum analysis of the final data set and cosmological implications, Monthly Notices of the Royal Astronomical Society, 362 (2005), 505-534.

[125] A.G. Sanchez et al., Cosmological parameters from cosmic microwave background measurements and the final $2 \mathrm{dF}$ Galaxy Redshift Survey power spectrum, Monthly Notices of the Royal Astronomical Society, 366 (2006), 189-207.

[126] M. Hicken et al., Improved Dark Energy Constraints from 100 New CfA Supernova Type Ia Light Curves, The Astrophysical Journal, 700 (2009), 1097-1140.

[127] E. Rozo et al., Cosmological Constraints from the Sloan Digital Sky Survey maxBCG Cluster Catalog, Astrophysical J., 708 (2010), 645-660.

[128] H. Lampeitl et al., First-year Sloan Digital Sky Survey-II supernova results: consistency and constraints with other intermediate-redshift data sets, Monthly Notices of the Royal Astronomical Society, 401 (2010), 2331-2342.

[129] A. Hajian et al., Measuring the thermal Sunyaev-Zel'dovich effect through the cross correlation of Planck and WMAP maps with ROSAT galaxy cluster catalogs, Journal of Cosmology and Astroparticle Physics, 11 (2013), 064:1-064:30.

[130] C. Blake et al., The WiggleZ Dark Energy Survey: measuring the cosmic expansion history using the Alcock-Paczynski test and distant supernovae, Monthly Notices of the Royal Astronomical Society, 418 (2011a), 1725-1735.

[131] R. Kessler et al., First-Year Sloan Digital Sky Survey-II Supernova Results: Hubble Diagram and Cosmological Parameters, Astrophysical J. Supplement, 185 (2009), 32-84.

[132] W.L. Percival et al., Baryon acoustic oscillations in the Sloan Digital Sky Survey Data Release 7 galaxy sample, Monthly Notices of the Royal Astronomical Society, 401 (2010), 2148-2168.

[133] A. Cabre and E. Gaztañaga, Clustering of luminous red galaxies - II. Small-scale redshift-space distortions, Monthly Notices of the Royal Astronomical Society, 396 (2009), 1119-1131.

[134] S. Cao, G. Covone, and Z.-H. Zhu, Testing the Dark Energy with Gravitational Lensing Statistics, Astrophys. J., 755 (2012), 31:1-31:13.

[135] A. Rest et al., Cosmological Constraints from Measurements of Type Ia Supernovae discovered during the first 1.5 years of the Pan-STARRS1 Survey, arXiv:, 1310.3828 (2013). 
[136] S.H. Suyu et al., Two Accurate Time-delay Distances from Strong Lensing: Implications for Cosmology, Astrophys.J., 766 (2013), 70:1-70:19.

[137] A. Mantz, S.W. Allen, D. Rapetti and H. Ebeling, The observed growth of massive galaxy clusters - I. Statistical methods and cosmological constraints, Monthly Notices of the Royal Astronomical Society, 406, (2010), 1759-1772.

[138] A. Vikhlinin et al., Chandra Cluster Cosmology Project III: Cosmological Parameter Constraints, Astrophys. J., 692 (2009), 1060-1074.

[139] H. Campbell et al., Cosmology with Photometrically Classified Type Ia Supernovae from the SDSS-II Supernova Survey, Astrophys. J., 763 (2013), 88:1-88:28.

[140] S. Ho, C. Hirata, N. Padmanabhan, U. Seljak and N. Bahcall, Correlation of CMB with large-scale structure. I. Integrated Sachs-Wolfe tomography and cosmological implications, Physical Review D, 78 (2008), 043519:1-043519:35.

[141] A. Del Popolo, V. Costa and G. Lanzafame, Improvements in the X-ray luminosity function and constraints on the cosmological parameters from X-ray luminous clusters, Astronomy and Astrophysics, 514 (2010), A80:1-A80:8.

[142] Q.-J. Zhang and Y.-L. Wu, Dark Energy and Hubble Constant From the Latest SNe Ia, BAO and SGL, arXiv: 0905.1234 (2009).

[143] B.A. Reid et al., Cosmological constraints from the clustering of the Sloan Digital Sky Survey DR7 luminous red galaxies, Monthly Notices of the Royal Astronomical Society, 404 (2010), 60-85.

[144] A.G. Sanchez, M. Crocce, A. Cabre, C.M. Baugh and E. Gaztañaga, Cosmological parameter constraints from SDSS luminous red galaxies: a new treatment of large-scale clustering, Monthly Notices of the Royal Astronomical Society, 400 (2009), 1643-1664.

[145] K.T. Mehta, A.J. Cuesta, X. Xu, D.J. Eisenstein and N. Padmanabhan, A 2 per cent distance to $\mathrm{z}=0.35$ by reconstructing baryon acoustic oscillations - III. Cosmological measurements and interpretation, Monthly Notices of the Royal Astronomical Society, 427 (2012), 2168-2179.

[146] M. Tegmark et al., Cosmological constraints from the SDSS luminous red galaxies, Physical Review D, 74 (2006), 123507:1-123507:36.

[147] D.J. Eisenstein et al., Detection of the Baryon Acoustic Peak in the Large-Scale Correlation Function of SDSS Luminous Red Galaxies, Astrophysical Journal, 633 (2005), 560-574.

[148] T. Okumura et al., Large-Scale Anisotropic Correlation Function of SDSS Luminous Red Galaxies, Astrophys. J., 676 (2008), 889-898.

[149] J.E. Taylor et al., Measuring the Geometry of the Universe from Weak Gravitational Lensing behind Galaxy Groups in the HST COSMOS Survey, Astrophys. J., 749 (2012), 127:1-127:12.

[150] S.W. Allen et al., Improved constraints on dark energy from Chandra X-ray observations of the largest relaxed galaxy clusters, Monthly Notices of the Royal Astronomical Society, 383 (2008), 879-896. 
[151] R. Mandelbaum et al., Cosmological parameter constraints from galaxy-galaxy lensing and galaxy clustering with the SDSS DR7, Monthly Notices of the Royal Astronomical Society, 432 (2013), 1544-1575.

[152] A. Cabre, E. Gaztañaga, M. Manera, P. Fosalba and F. Castander, Cross-correlation of Wilkinson Microwave Anisotropy Probe third-year data and the Sloan Digital Sky Survey DR4 galaxy survey: new evidence for dark energy, Monthly Notices of the Royal Astronomical Society: Letters, 372 (2006), L23-L27.

[153] C. Blake et al., The WiggleZ Dark Energy Survey: mapping the distance-redshift relation with baryon acoustic oscillations, Monthly Notices of the Royal Astronomical Society, 418 (2011b), 1707-1724.

[154] W.L. Freedman et al., The Carnegie Supernova Project: First Near-Infrared Hubble Diagram to $z \sim 0.7$, Astrophysical Journal, 704 (2009), 1036-1058.

[155] W.M. Wood-Vasey et al., Observational Constraints on the Nature of Dark Energy: First Cosmological Results from the ESSENCE Supernova Survey, Astrophysical Journal, 666 (2007), 694-715.

[156] M. Sullivan et al., SNLS3: Constraints on Dark Energy Combining the Supernova Legacy Survey Three-year Data with Other Probes, Astrophys. J., 737 (2011), 102:1102:19.

[157] K.M. Wilson, G. Chen and B. Ratra, Supernova Ia and Galaxy Cluster Gas Mass Fraction Constraints on Dark Energy, Modern Physics Letters A, 21 (2006), 219-2204.

[158] M. Oguri et al., The Sloan Digital Sky Survey Quasar Lens Search. VI. Constraints on Dark Energy and the Evolution of Massive Galaxies, Astron. J., 143 (2012), 120:1-120:14.

[159] G. Hinshaw et al., Nine-year Wilkinson Microwave Anisotropy Probe (WMAP) Observations: Cosmological Parameter Results, Astrophys. J. Suppl., 208 (2013), 19:1-19:25.

[160] S. Cao, and Z.-H. Zhu, Constraints on cosmological models from lens redshift data, Astronomy and Astrophysics, 538 (2012), A43:1-A43:7.

[161] L.D. Ferramacho, A. Blanchard and Y. Zolnierowski, Constraints on CDM cosmology from galaxy power spectrum, CMB and SNIa evolution, Astronomy and Astrophysics, 499 (2009), 21-29.

[162] A. Clocchiatti et al., Hubble Space Telescope and Ground-based Observations of Type Ia Supernovae at Redshift 0.5: Cosmological Implications, Astrophysical Journal, 642 (2006), 1-21.

[163] D. Parkinson et al., The WiggleZ Dark Energy Survey: Final data release and cosmological results, Physical Review D, 86 (2012), 103518:1- 103518:23.

[164] L. Samushia et al., The clustering of galaxies in the SDSS-III DR9 Baryon Oscillation Spectroscopic Survey: testing deviations from Lambda and general relativity using anisotropic clustering of galaxies, Monthly Notices of the Royal Astronomical Society, 429 (2013), 1514-1528.

[165] E.M. Huff et al., Seeing in the dark - II. Cosmic shear in the Sloan Digital Sky Survey, arXiv:1112.3143 (2011), 1-23. 
[166] A. Del Popolo, Constraints on the cosmological parameters by means of the clusters mass function, Memorie della Societa Astronomica Italiana, 81 (2010), 157-162.

[167] N.P. Ross et al., The 2dF-SDSS LRG and QSO Survey: the LRG 2-point correlation function and redshift-space distortions, Monthly Notices of the Royal Astronomical Society, 381 (2007), 573-588.

[168] C. Blake et al., The WiggleZ Dark Energy Survey: small-scale clustering of Lymanbreak galaxies at z $<1$, Monthly Notices of the Royal Astronomical Society, 395 (2009), 240-254.

[169] E. Gaztañaga, Dark Energy from the milimeter sky, Revista Mexicana de Astronomia y Astrofisica (Serie de Conferencias), 24 (2005), 40-44.

[170] P. Astier et al., The Supernova Legacy Survey: measurement of $\Omega_{M}, \Omega_{\Lambda}$ and $w$ from the first year data set, Astronomy and Astrophysics, 447 (2006), 31-48.

[171] B.A. Reid et al., The clustering of galaxies in the SDSS-III Baryon Oscillation Spectroscopic Survey: measurements of the growth of structure and expansion rate at $\mathrm{z}=$ 0.57 from anisotropic clustering, Monthly Notices of the Royal Astronomical Society, 426 (2012), 2719-2737.

[172] C.-H. Chuang et al., The clustering of galaxies in the SDSS-III Baryon Oscillation Spectroscopic Survey: single-probe measurements and the strong power of $f(z) \sigma_{8}(z)$ on constraining dark energy, Monthly Notices of the Royal Astronomical Society, 433 (2013), 3559-3571.

[173] G.-B. Zhao et al., The clustering of galaxies in the SDSS-III Baryon Oscillation Spectroscopic Survey: weighing the neutrino mass using the galaxy power spectrum of the CMASS sample, Monthly Notices of the Royal Astronomical Society, 436 (2013), 20382053.

[174] L. Anderson et al., The clustering of galaxies in the SDSS-III Baryon Oscillation Spectroscopic Survey: baryon acoustic oscillations in the Data Release 9 spectroscopic galaxy sample, Monthly Notices of the Royal Astronomical Society, 427 (2012), 3435-3467.

[175] Y. Chen, C.-Q. Geng, S. Cao, Y.-M. Huang and Z.-H. Zhu, Constraints on the inverse power-law scalar field dark energy model from strong gravitational lensing data and updated Hubble parameter measurements, arXiv: 1312.1443 (2013).

[176] O. Farooq and B. Ratra, Hubble Parameter Measurement Constraints on the Cosmological Deceleration-Acceleration Transition Redshift, Astrophys. J. Lett., 766 (2013), L7:1L7:4.

[177] J. Guy et al., The Supernova Legacy Survey 3-year sample: Type Ia supernovae photometric distances and cosmological constraints, Astronomy and Astrophysics, 523 (2010), A7:1-A7:34.

[178] A. Feoli, L. Mancini, V. Rillo and M. Grasso, Cosmological constraints from supernova data set with corrected redshift, Journal of Physics: Conference Series, 354 (2012), 012005:1-012005:10.

[179] A. Conley et al., Supernova Constraints and Systematic Uncertainties from the First Three Years of the Supernova Legacy Survey, Astrophys. J. Supplement, 192 (2011), 1:11:29. 
[180] G.E. Addison, G. Hinshaw and M. Halpern, Cosmological constraints from baryon acoustic oscillations and clustering of large-scale structure, Monthly Notices of the Royal Astronomical Society, 436 (2013), 1674-1683.

[181] M. Ganeshalingam, W. Li and A.V. Filippenko, Constraints on dark energy with the LOSS SN Ia sample, Monthly Notices of the Royal Astronomical Society, 433 (2013), 2240-2258.

[182] H. Lin et al., The SDSS Co-add: Cosmic Shear Measurement, Astrophys. J., 761 (2012), $15: 1-15: 15$.

[183] S. Carneiro, C. Pigozzo, H.A. Borges and J.S. Alcaniz, Supernova constraints on decaying vacuum cosmology, Physical Review D, 74 (2006), 023532:1-023532:7.

[184] T.M. Davis et al., Scrutinizing Exotic Cosmological Models Using ESSENCE Supernova Data Combined with Other Cosmological Probes, Astrophys. J., 666 (2007), 716725.

[185] M. Sereno and D. Paraficz, Hubble constant and dark energy inferred from free-form determined time delay distances, Monthly Notices of the Royal Astronomical Society, 437 (2014), 600-605.

[186] C. Heymans et al., CFHTLenS tomographic weak lensing cosmological parameter constraints: Mitigating the impact of intrinsic galaxy alignments, Monthly Notices of the Royal Astronomical Society, 432 (2013), 2433-2453.

[187] U. Seljak et al., Cosmological parameter analysis including SDSS Ly alpha forest and galaxy bias: Constraints on the primordial spectrum of fluctuations, neutrino mass, and dark energy, Physical Review D, 71 (2005), 103515:1-103515:21.

[188] W.L. Freedman et al., Carnegie Hubble Program: A Mid-infrared Calibration of the Hubble Constant, Astrophysical Journal, 758 (2012), 24:1-24:10.

[189] B.A. Benson et al., Cosmological Constraints from Sunyaev-Zel'dovich-selected Clusters with X-Ray Observations in the First $178 \mathrm{deg}^{2}$ of the South Pole Telescope Survey, Astrophysical J., $\mathbf{7 6 3}$ (2013), 147:1-147:21.

[190] M.C. March, R. Trotta, P. Berkes, G.D. Starkman and P.M. Vaudrevange, Improved constraints on cosmological parameters from Type Ia supernova data, Monthly Notices of the Royal Astronomical Society, 418 (2011), 2308-2329.

[191] J. Benjamin et al., Cosmological constraints from the 100- $\mathrm{deg}^{2}$ weak-lensing survey, Monthly Notices of the Royal Astronomical Society, 381 (2007), 702-712.

[192] S. Ettori et al., The cluster gas mass fraction as a cosmological probe: a revised study, Astronomy and Astrophysics, 501 (2009), 61-73.

[193] T. Giannantonio et al., Combined analysis of the integrated Sachs-Wolfe effect and cosmological implications, Physical Review D, 77 (2008), 123520:1-123520:24.

[194] Y. Chen and B. Ratra, Hubble parameter data constraints on dark energy, Physics Letters $B, 703$ (2011), 406-411.

[195] E. Semboloni et al., Cosmic shear analysis with CFHTLS deep data, Astronomy and Astrophysics, 452 (2006), 51-61. 
[196] L. Fu et al., Very weak lensing in the CFHTLS wide: cosmology from cosmic shear in the linear regime, Astronomy and Astrophysics, 479 (2008), 9-25.

[197] H.K. Jassal, J.S. Bagla and T. Padmanabhan, Understanding the origin of CMB constraints on dark energy, Monthly Notices of the Royal Astronomical Society, 405 (2010), 2639-2650.

[198] C. Firmani, V. Avila-Reese, G. Ghisellini and G. Ghirlanda, The Hubble diagram extended to $z \gg 1$ : the gamma-ray properties of gamma-ray bursts confirm the Lambda cold dark matter model, Monthly Notices of the Royal Astronomical Society: Letters, 372 (2006), L28-L32.

[199] S. Basilakos and M. Plionis, Breaking the $\sigma_{8}-\Omega_{m}$ Degeneracy Using the Clustering of High-z X-ray Active Galactic Nuclei, Astrophys.J.Lett., 714 (2010), L185-L189.

[200] N. Suzuki et al., The Hubble Space Telescope Cluster Supernova Survey. V. Improving the Dark-energy Constraints above z $>1$ and Building an Early-type-hosted Supernova Sample, Astrophys. J., 746 (2012), 85:1-85:24.

[201] J.-J. Wei, X.-F Wu, and F. Melia, The Gamma-Ray Burst Hubble Diagram and Its Implications for Cosmology, Astrophysical J., 772 (2013), 43:1-43:11.

[202] M. Lopez-Corredoira, Alcock-Paczynski cosmological test, arXiv: 1312.0003 (2013).

[203] N. Suzuki et al., The Hubble Space Telescope Cluster Supernova Survey. V. Improving the Dark-energy Constraints above $\mathrm{z}>1$ and Building an Early-type-hosted Supernova Sample, Astrophys. J., 746 (2012), 85:1-85:24.

[204] J. da Angela et al., The 2dF QSO Redshift Survey- XV. Correlation analysis of redshiftspace distortions, Monthly Notices of the Royal Astronomical Society, 360 (2005a), 10401054.

[205] J. da Angela et al., The 2dF-SDSS LRG and QSO survey: QSO clustering and the L-Z degeneracy, Monthly Notices of the Royal Astronomical Society, 383 (2008), 565-580.

[206] Y. Kodama et al., Gamma-ray bursts in $1.8<\mathrm{z}<5.6$ suggest that the time variation of the dark energy is small, Monthly Notices of the Royal Astronomical Society: Letters, 391 (2008), L1-L4.

[207] R. Tsutsui et al., Constraints on $w_{0}$ and $w_{a}$ of dark energy from high-redshift gamma-ray bursts, Monthly Notices of the Royal Astronomical Society: Letters, 394 (2009), L31-L35.

[208] N.G. Busca et al., Baryon acoustic oscillations in the Ly $\alpha$ forest of BOSS quasars, Astronomy and Astrophysics, 552 (2013, A96:1-A96:18.

[209] V.C. Busti, R.C. Santos and J.A.S. Lima, Constraining the dark energy and smoothness parameter with type Ia supernovae and gamma-ray bursts, Physical Review D, 85 (2012), 103503:1-103503:7.

[210] J. da Angela, P.J. Outram and T. Shanks, Constraining beta(z) and $\Omega_{m}^{0}$ from redshiftspace distortions in $z \sim 3$ galaxy surveys, Monthly Notices of the Royal Astronomical Society, 361 (2005b), 879-886.

[211] J. Dunkley et al., Five-Year Wilkinson Microwave Anisotropy Probe Observations: Likelihoods and Parameters from the WMAP Data, Astrophysical J. Supplement, 180 (2009), 306-329. 
[212] Planck Collaboration; P.A.R. Ade et al., Planck 2013 results. XVI. Cosmological parameters, arXiv: $\mathbf{1 3 0 3 . 5 0 7 6}$ (2013).

[213] C.L. Bennett et al., Nine-year Wilkinson Microwave Anisotropy Probe (WMAP) Observations: Final Maps and Results, Astrophysical Journal Supplement, 208 (2013), 20:120:54.

[214] D.N. Spergel et al., Three-Year Wilkinson Microwave Anisotropy Probe (WMAP) Observations: Implications for Cosmology, Astrophysical Journal Supplement Series, 170 (2007), 377-408.

[215] C.L. Reichardt et al., High-Resolution CMB Power Spectrum from the Complete ACBAR Data Set, Astrophys. J., 694 (2009), 1200-1219.

[216] E. Komatsu et al., Five-Year Wilkinson Microwave Anisotropy Probe Observations: Cosmological Interpretation, Astrophys. J. Suppl., 180 (2009), 330-376.

[217] E. Komatsu et al., Seven-year Wilkinson Microwave Anisotropy Probe (WMAP) Observations: Cosmological Interpretation, Astrophysical J. Suppl., 192 (2011), 18:1-18:47.

[218] Y. Wang, Model-independent distance measurements from gamma-ray bursts and constraints on dark energy, Physical Review D, 78 (2008), 123532:1-123532:8.

[219] N. Liang, W.K. Xiao, Y. Liu and S.N. Zhang, A Cosmology-Independent Calibration of Gamma-Ray Burst Luminosity Relations and the Hubble Diagram, Astrophys.J., 685 (2008), 354-360.

[220] B.E. Schaefer, The Hubble Diagram to Redshift > 6 from 69 Gamma-Ray Bursts, Astrophys. J., 660 (2007), 16-46.

[221] B.D. Sherwin et al., Evidence for Dark Energy from the Cosmic Microwave Background Alone Using the Atacama Cosmology Telescope Lensing Measurements, Physical Review Letters, 107 (2011), 021302:1-021302:5.

[222] D. Pietrobon, A. Balbi and D. Marinucci, Integrated Sachs-Wolfe effect from the cross correlation of WMAP 3year and the NRAO VLA sky survey data: New results and constraints on dark energy, Physical Review D, 74 (2006), 043524:1-043524:9.

[223] D. Spergel, R. Flauger and R. Hlozek, Planck Data Reconsidered,arXiv: 1312.3313 (2013), 1-15.

[224] J.L. Sievers et al., The Atacama Cosmology Telescope: cosmological parameters from three seasons of data, Journal of Cosmology and Astroparticle Physics, 10 (2013), 060:1060:26.

[225] D. Larson et al., Seven-year Wilkinson Microwave Anisotropy Probe (WMAP) Observations: Power Spectra and WMAP-derived Parameters, Astrophysical Journal Supplement, 192 (2011), 16:1-16:19.

[226] C. M. Will, The Confrontation between General Relativity and Experiment, Living Reviews in Relativity, 9 (2006), 3:1-3:100.

[227] N. Yunes and X. Siemens, Gravitational-Wave Tests of General Relativity with GroundBased Detectors and Pulsar-Timing Arrays, Living Reviews in Relativity, 16 (2019), 9:19:124. 
[228] J. R. Gair, M. Vallisneri, S. L. Larson and J. G. Baker, Testing General Relativity with Low-Frequency, Space-Based Gravitational-Wave Detectors, Living Reviews in Relativity, 16 (2013) 7:1-7:109.

[229] K. S. Thorne and J. B. Hartle, Laws of motion and precession for black holes and other bodies, Rev.Mod.Phys.D, 31 (1985), 1815-1837.

[230] K. S. Thorne, R. M. Price and D. A. Macdonald, Black Holes: The Membrane Paradigm, (Yale Univ. Press, New Haven, 1986).

[231] N. Wex and S. M. Kopeikin, Frame dragging and other precessional effects in black hole pulsar binaries, Astrophys.J., 514 (1999), 388-401

[232] C. M. Will, Testing the general relativistic "no-hair" theorems using the Galactic Center black hole Sagittarius A*, Astrophys.J., 674 (2008), L25-L28.

[233] A. Sillanpää, S. Haarala, M. J. Valtonen, B. Sundelius and G. G. Byrd, OJ287 - Binary pair of supermassive black holes, Astrophys.J., 325 (1988), 628-634.

[234] M. J. Valtonen et al., Predicting the next outbursts of OJ287 in 2006-2010, Astrophys.J., 646 (2006), 36-48.

[235] P. Pihajoki, M. Valtonen and S. Ciprini, Short time-scale periodicity in OJ287, Mon.Not.RAS, 434 (2013), 3122-3129.

[236] M. J. Valtonen, S. Mikkola, H. J. Lehto, A. Gopakumar, R. Hudec and J. Polednikova, Testing the Black Hole No-hair Theorem with OJ287, Astrophys.J., 742 (2011a), 22-33.

[237] L. Barack and C. Cutler, Using LISA extreme-mass-ratio inspiral sources to test offKerr deviations in the geometry of massive black holes, Physical Review D, 75 (2007), 042003:1-042003:11.

[238] A.E. Broderick, T. Johannsen, A. Loeb and D. Psaltis, Testing the No-Hair Theorem with Event Horizon Telescope Observations of Sagittarius A*, arXiv: 1311.5564 (2013)

[239] T. Johannsen and D. Psaltis, Testing the No-hair Theorem with Observations in the Electromagnetic Spectrum. I. Properties of a Quasi-Kerr Spacetime, Astrophys.J., 716 (2010a), 187-197.

[240] T. Johannsen and D. Psaltis, Testing the No-hair Theorem with Observations in the Electromagnetic Spectrum. II. Black Hole Images, Astrophys.J., 718 (2010b), 446-454.

[241] T. Johannsen and D. Psaltis, Testing the No-hair Theorem with Observations in the Electromagnetic Spectrum. III. Quasi-periodic Variability, Astrophys.J., 726 (2011), 1120.

[242] T. Johannsen and D. Psaltis, Testing the No-hair Theorem with Observations in the Electromagnetic Spectrum. IV. Relativistically Broadened Iron Lines, Astrophys.J., 773 (2013), 57-65.

[243] C. Bambi and E. Barausse, Final stages of accretion onto non-Kerr compact objects, Physical Review D, 84 (2011), 084034:1-084034:13.

[244] C. Bambi and E. Barausse, Constraining the Quadrupole Moment of Stellar-mass Black Hole Candidates with the Continuum Fitting Method, The Astrophysical Journal, 731 (2011b), 121:1-121:12. 
[245] C. Bambi, Towards the use of the most massive black hole candidates in active galactic nuclei to test the Kerr paradigm, Physical Review D, vol. 85 (2012a), 043001:1043001:11.

[246] C. Bambi, A Code to Compute the Emission of Thin Accretion Disks in Non-Kerr Spacetimes and Test the Nature of Black Hole Candidates, The Astrophysical Journal, Volume 761 (2012b), 174:1-174:9.

[247] C. Bambi, Probing the space-time geometry around black hole candidates with the resonance models for high-frequency QPOs and comparison with the continuum-fitting method, Journal of Cosmology and Astroparticle Physics, 09 (2012c), 014:1-014:17.

[248] C. Bambi, Testing the space-time geometry around black hole candidates with the analysis of the broad K $\alpha$ iron line, Physical Review D, 87 (2013), 023007:1-023007:12.

[249] H. Krawczynski, Tests of General Relativity in the Strong-gravity Regime Based on X-Ray Spectropolarimetric Observations of Black Holes in X-Ray Binaries, The Astrophysical Journal, 754 (2012), 133:1-133:12.

[250] M. J. Valtonen, M. Kidger, H. Lehto and G. Poyner, The structure of the October/November 2005 outburst in OJ287 and the precessing binary black hole model, Astronomy and Astrophysics, 477 (2008a), 407-412.

[251] M. J. Valtonen, OJ287: a binary black hole system, Rev. Mex. Astron. y Astrofis. (Ser. Conf., The Nuclear Region, Host Galaxy and Environment of Active Galaxies, Huatulco, Mexico, April 18-20, 2007), 32 (2008), 22-24.

[252] M. J. Valtonen et al., A massive binary black-hole system in OJ287 and a test of general relativity, Nature, $\mathbf{4 5 2}$ (2008b), 851-853.

[253] H. J. Lehto and M. J. Valtonen, OJ287 outburst structure and a binary black hole model, Astrophys.J., 460 (1996), 207-213.

[254] M. J. Valtonen and P. Pihajoki, A helical jet model for OJ287, Astronomy and Astrophysics, 557 (2013), A28:1-A38:5.

[255] B. Sundelius, M. Wahde, H. J. Lehto and M. J. Valtonen, A numerical simulation of the brightness variations of OJ287, Astrophys.J., 484 (1997), 180-185.

[256] P. B. Ivanov, I. V. Igumenshchev and I. D. Novikov, Hydrodynamics of Black HoleAccretion Disk Collision, Astrophys.J., 507 (1998), 131-144.

[257] M. J. Valtonen, S. Ciprini and H. J. Lehto, On the masses of OJ287 black holes, Mon.Not.RAS, 427 (2012), 77-83.

[258] M. J. Valtonen, New orbit solutions for the precessing binary black hole model of OJ287, Astrophys.J., 659 (2007), 1074-1081.

[259] N. I. Shakura and R. A. Sunyaev, Black holes in binary systems. Observational appearance, Astronomy and Astrophysics, 24 (1973), 337-355.

[260] P. J. Sakimoto and F. V. Corotini, Accretion disk models for QSOs and active galactic nuclei - The role of magnetic viscosity, Astrophys.J., 247 (1981), 19-31.

[261] A. R. King, J. E. Pringle and M. Livio, Accretion disc viscosity: how big is alpha?, Mon.Not.RAS, 376 (2007), 1740-1746. 
[262] M. J. Valtonen, H. J. Lehto, L. O. Takalo and A. Sillanpää, Testing the 1995 Binary Black Hole Model of OJ287, Astrophys.J., 729 (2011b), 33-38.

[263] M. J. Valtonen and K. Wiik, Optical polarization angle and VLBI jet direction in the binary black hole model of OJ287, Mon.Not.RAS, 421 (2012), 1861-1867.

[264] H. Baumgardt, A. Gualandris and S. Portegies Zwart, Ejection of hypervelocity stars from the Galactic Centre by intermediate-mass black holes, Mon.Not.RAS, 372 (2006), $174-182$.

[265] T. Matsubayashi, J. Makino and T. Ebisuzaki, Orbital evolution of an IMBH in the Galactic Nucleus with a Massive Central Black Hole, Astrophys.J., 656 (2007), 879-896.

[266] M. Iwasawa, S. An, T. Matsubayashi, Y. Funato and J. Makino, Eccentric Evolution of Supermassive Black Hole Binaries, Astrophys.J.(Lett.), 731 (2011), L9-L13.

[267] M. J. Valtonen et al., Measuring the Spin of the Primary Black Hole in OJ287, Astrophys.J., 709 (2010a), 725-732.

[268] L. E. Kidder, Coalescing binary systems of compact objects to (post)5/2-Newtonian order. V. Spin effects, Physical Review D, 52 (1995), 821-847.

[269] T. Mora and C. M. Will, Post-Newtonian diagnostic of quasiequilibrium binary configurations of compact objects, Physical Review D, 69 (2004), 104021:1-104021:25.

[270] S. Kumar and J. E. Pringle, Twisted accretion discs - The Bardeen-Petterson effect, Mon.Not.RAS, 213 (1985), 435-442.

[271] G. Lodato and J. E. Pringle, The evolution of misaligned accretion discs and spinning black holes, Mon.Not.RAS, 368 (2006), 1196-1208.

[272] R. Hudec, M. Basta, P. Pihajoki and M. Valtonen, The historical 1900 and 1913 outbursts of the binary blazar candidate OJ287, Astronomy and Astrophysics, 559 (2013), A20:1-A20:9.

[273] M. J. Valtonen, The OJ287 binary model and the expected outburst in November 1995, Tuorla Obs.Rep. (Workshop on Two Years of Intensive Monitoring of OJ287 and 3C66A, Oxford, England, September 11-14, 1995), 176 (1996), 64-72.

[274] M. J. Valtonen et al., Tidally Induced Outbursts in OJ 287 during 2005-2008, Astrophys.J., 698 (2009), 781-785.

\section{Author information}

Gene G. Byrd, University of Alabama, Tuscaloosa, AL, 35487-0324, USA.

E-mail: genebyrd@bellsouth. net

Arthur Chernin, Sternberg Astronomical Institute, Moscow University, 119899, Moscow,

Russia.

E-mail: arthur .chernin@gmail.com 
Pekka Teerikorpi, Tuorla Observatory, Department of Physics and Astronomy, University of Turku, 21500, Piikkiö, Finland.

E-mail: pekkatee@utu.fi

Mauri Valtonen, FINCA, University of Turku, FI-21500 Piikkiö, Finland.

E-mail: mvaltonen2001@yahoo.com 
Table $1 . \Omega_{\Lambda}$ determinations at different redshifts.

\begin{tabular}{|c|c|c|c|}
\hline Redshift & $\Omega_{\Lambda}$ & Uncertainty & Reference \\
\hline 0 & 0.75 & 0.07 & this work \\
\hline 0 & 0.71 & 0.07 & Chernin (2013) \\
\hline 0 & 0.76 & 0.07 & Karachentsev et al. (2009) \\
\hline 0.005 & 0.78 & 0.08 & Mohayaee and Tully (2005) \\
\hline 0.01 & 0.70 & 0.14 & Ma and Scott (2013) \\
\hline 0.01 & 0.76 & 0.13 & Nusser and Davis (2011) \\
\hline 0.01 & 0.71 & 0.10 & Park and Park (2006) \\
\hline 0.05 & 0.71 & 0.05 & Kowalski et al. (2008) \\
\hline 0.06 & 0.68 & 0.10 & Henry et al. (2009) \\
\hline 0.07 & 0.75 & 0.02 & Beutler et al. (2012) \\
\hline 0.1 & 0.71 & 0.03 & Tinker et al. (2012) \\
\hline 0.1 & 0.71 & 0.03 & Simha and Cole (2013) \\
\hline 0.1 & 0.72 & 0.03 & Cacciato et al. (2013) \\
\hline 0.1 & 0.76 & 0.02 & Cole et al. (2005) \\
\hline 0.1 & 0.78 & 0.02 & Sanchez et al. (2006) \\
\hline 0.16 & 0.72 & 0.08 & Hicken et al. (2009) \\
\hline 0.2 & 0.72 & 0.07 & Rozo et al. (2010) \\
\hline 0.2 & 0.78 & 0.08 & Lampeitl et al. (2010) \\
\hline 0.2 & 0.72 & 0.02 & Hajian et al. (2013) \\
\hline 0.22 & 0.72 & 0.05 & Blake et al. (2011a) \\
\hline 0.23 & 0.73 & 0.03 & Kessler et al. (2009) \\
\hline 0.275 & 0.71 & 0.02 & Percival et al. (2010) \\
\hline 0.3 & 0.75 & 0.05 & Cabre and Caztañaga (2009) \\
\hline 0.3 & 0.75 & 0.07 & Cao et al. (2012) \\
\hline 0.3 & 0.80 & 0.15 & Rest et al. (2013) \\
\hline 0.3 & 0.75 & 0.10 & Suyu et al. (2013) \\
\hline 0.3 & 0.78 & 0.06 & Mantz et al. (2010) \\
\hline 0.3 & 0.75 & 0.08 & Vikhlinin et al. (2009) \\
\hline 0.3 & 0.76 & 0.06 & Campbell et al. (2013) \\
\hline 0.3 & 0.75 & 0.06 & Ho et al. (2008) \\
\hline 0.3 & 0.73 & 0.02 & Del Popolo et al. (2010) \\
\hline 0.35 & 0.64 & 0.12 & Zhang and Wu (2009) \\
\hline 0.35 & 0.72 & 0.04 & Reid et al. (2010) \\
\hline 0.35 & 0.74 & 0.02 & Sanchez et al. (2009) \\
\hline 0.35 & 0.72 & 0.02 & Mehta et al. (2012) \\
\hline 0.35 & 0.76 & 0.04 & Tegmark et al. (2006) \\
\hline 0.35 & 0.73 & 0.04 & Eisenstein et al. (2005) \\
\hline
\end{tabular}


Table 2. $\Omega_{\Lambda}$ table continued

\begin{tabular}{|c|c|c|c|}
\hline Redshift & $\Omega_{\Lambda}$ & Uncertainty & Reference \\
\hline 0.36 & 0.77 & 0.05 & Okumura et al. (2008) \\
\hline 0.4 & 0.85 & 0.08 & Taylor et al. (2012) \\
\hline 0.4 & 0.72 & 0.06 & Allen et al. (2008) \\
\hline 0.4 & 0.73 & 0.04 & Mandelbaum et al. (2013) \\
\hline 0.4 & 0.82 & 0.03 & Cabre et al. (2006) \\
\hline 0.4 & 0.71 & 0.09 & Blake et al. (2011b) \\
\hline 0.4 & 0.76 & 0.20 & Freedman et al. (2009) \\
\hline 0.41 & 0.71 & 0.07 & Blake et al. (2010) \\
\hline 0.43 & 0.73 & 0.04 & Wood-Vasey et al. (2007) \\
\hline 0.45 & 0.85 & 0.15 & Sullivan et al. (2011) \\
\hline 0.48 & 0.73 & 0.15 & Wilson et al. (2006) \\
\hline 0.5 & 0.79 & 0.12 & Oguri et al. (2012) \\
\hline 0.5 & 0.72 & 0.03 & Hinshaw et al. (2013) \\
\hline 0.5 & 0.85 & 0.15 & Cao and Zhu (2012) \\
\hline 0.5 & 0.74 & 0.04 & Ferramacho et al. (2009) \\
\hline 0.5 & 0.71 & 0.06 & Clocchiatti et al. (2006) \\
\hline 0.5 & 0.71 & 0.02 & Parkinson et al. (2012) \\
\hline 0.5 & 0.72 & 0.02 & Samushia et al. (2013) \\
\hline 0.52 & 0.75 & 0.10 & Huff et al. (2011) \\
\hline 0.55 & 0.75 & 0.10 & Del Popolo (2010) \\
\hline 0.55 & 0.70 & 0.15 & Ross et al. (2007) \\
\hline 0.55 & 0.73 & 0.04 & Blake et al. (2009) \\
\hline 0.5 & 0.70 & 0.10 & Caztañaga (2005) \\
\hline 0.55 & 0.73 & 0.04 & Astier et al. (2006) \\
\hline 0.57 & 0.74 & 0.05 & Reid et al. (2012) \\
\hline 0.57 & 0.74 & 0.04 & Chuang et al. (2013) \\
\hline 0.57 & 0.72 & 0.03 & Zhao et al. (2013) \\
\hline 0.57 & 0.71 & 0.02 & Anderson et al. (2012) \\
\hline 0.57 & 0.72 & 0.04 & Chen et al. (2013) \\
\hline 0.59 & 0.70 & 0.20 & Farooq and Ratra (2013) \\
\hline 0.6 & 0.69 & 0.13 & Blake et al. (2011a) \\
\hline 0.6 & 0.79 & 0.10 & Guy et al. (2010) \\
\hline 0.6 & 0.61 & 0.05 & Feoli et al. (2012) \\
\hline 0.6 & 0.75 & 0.10 & Conley et al. (2011) \\
\hline 0.6 & 0.70 & 0.04 & Addison et al. (2013) \\
\hline 0.6 & 0.64 & 0.12 & Ganeshalingam et al. (2013) \\
\hline 0.6 & 0.76 & 0.05 & Lin et al. (2011) \\
\hline
\end{tabular}


Table $3 . \Omega_{\Lambda}$ table continued

\begin{tabular}{|c|c|c|c|}
\hline Redshift & $\Omega_{\Lambda}$ & Uncertainty & Reference \\
\hline 0.6 & 0.68 & 0.05 & Carneiro et al. (2006) \\
\hline 0.65 & 0.73 & 0.04 & Davis et al. (2007) \\
\hline 0.66 & 0.71 & 0.02 & Sereno and Paraficz (2014) \\
\hline 0.7 & 0.84 & 0.10 & Heymans et al. (2013) \\
\hline 0.7 & 0.72 & 0.02 & Seljak et al. (2005) \\
\hline 0.7 & 0.75 & 0.02 & Freedman et al. (2012) \\
\hline 0.75 & 0.75 & 0.07 & Benson et al. (2013) \\
\hline 0.75 & 0.73 & 0.02 & March et al. (2011) \\
\hline 0.78 & 0.78 & 0.09 & Blake et al. (2011a) \\
\hline 0.78 & 0.76 & 0.15 & Benjamin et al. (2007) \\
\hline 0.80 & 0.65 & 0.10 & Ettori et al. (2009) \\
\hline 0.80 & 0.73 & 0.10 & Giannantonio et al. (2008) \\
\hline 0.88 & 0.72 & 0.08 & Chen and Ratra (2011) \\
\hline 0.9 & 0.68 & 0.08 & Semboloni et al. (2006) \\
\hline 0.95 & 0.75 & 0.05 & Fu et al. (2008) \\
\hline 1.0 & 0.73 & 0.05 & Jassal et al. (2010) \\
\hline 1.0 & 0.73 & 0.03 & Firmani et al. (2006) \\
\hline 1.0 & 0.76 & 0.06 & Basilakos and Plionis (2010) \\
\hline 1.02 & 0.73 & 0.02 & Suzuki et al. (2012) \\
\hline 1.3 & 0.75 & 0.06 & Wei et al. (2013) \\
\hline 1.3 & 0.76 & 0.09 & Lopez-Corredoira (2013) \\
\hline 1.3 & 0.73 & 0.02 & Suzuki et al. (2012) \\
\hline 1.4 & 0.65 & 0.15 & da Angela et al. (2005a) \\
\hline 1.5 & 0.75 & 0.08 & da Angela et al. (2008) \\
\hline 1.6 & 0.63 & 0.13 & Kodama et al. (2008) \\
\hline 1.6 & 0.64 & 0.10 & Tsutsui et al. (2009) \\
\hline 2.3 & 0.75 & 0.10 & Busca et al. (2013) \\
\hline 2.5 & 0.73 & 0.06 & Busti et al. (2012) \\
\hline 3 & 0.65 & 0.20 & Da Angela et al. (2005b) \\
\hline 3 & 0.75 & 0.03 & Dunkley et al. (2009) \\
\hline 3 & 0.69 & 0.02 & The Planck Collaboration: Ade et al. (2013) \\
\hline 3 & 0.72 & 0.03 & Bennett et al. (2013) \\
\hline 3 & 0.76 & 0.02 & Spergel et al. (2007) \\
\hline 3 & 0.74 & 0.03 & Reichardt et al. (2009) \\
\hline 3 & 0.73 & 0.02 & Komatsu et al. (2009) \\
\hline 3 & 0.73 & 0.02 & Komatsu et al. (2011) \\
\hline 3 & 0.75 & 0.12 & Wang (2008) \\
\hline
\end{tabular}


Table 4. $\Omega_{\Lambda}$ table continued

\begin{tabular}{l|r|r|l}
\hline \hline Redshift & $\Omega_{\Lambda}$ & Uncertainty & Reference \\
\hline 3 & 0.75 & 0.05 & Liang et al. (2008) \\
3 & 0.73 & 0.12 & Schaefer (2007) \\
3 & 0.61 & 0.14 & Sherwin et al. (2011) \\
3 & 0.65 & 0.15 & Pietrobon et al. (2006) \\
3 & 0.70 & 0.02 & Spergel et al. (2013) \\
3 & 0.65 & 0.10 & Sievers et al. (2013) \\
3 & 0.74 & 0.02 & Larson et al. (2011)
\end{tabular}

Table 5. Solution parameters.

\begin{tabular}{l|r|r}
\hline \hline Parameter & Astrophysics & Orbit \\
\hline$\Delta \phi$ & $38 .{ }^{\circ} \pm \pm 1.0$ & $39 .{ }^{\circ} 1 \pm 0 .{ }^{\circ} 1$ \\
$m_{1}$ & $(1.8 \pm 0.1) \cdot 10^{10} M_{\odot}$ & $(1.84 \pm 0.01) \cdot 10^{10} M_{\odot}$ \\
$m_{2}$ & $(1.4 \pm 0.4) \cdot 10^{8} M_{\odot}$ & $(1.46 \pm 0.1) \cdot 10^{8} M_{\odot}$ \\
$\chi_{1}$ & $0.25 \pm 0.04$ & $0.28 \pm 0.03$ \\
$\phi_{0}$ & $56.0 \pm 4.0$ & $56.3 \pm 1.0$ \\
$e$ & $0.7 \pm 0.03$ & $0.70 \pm 0.001$ \\
$\alpha$ & $0.3 \pm 0.2$ & $0.3 \pm 0.1$ \\
$q$ & - & $1.0 \pm 0.3$
\end{tabular}

Table 6. Outburst times with estimated uncertainties. These are starting times of the outbursts.

\begin{tabular}{lr}
\hline \hline Time & uncertainty \\
\hline 1912.970 & \pm 0.010 \\
1947.282 & \pm 0.0005 \\
1957.080 & \pm 0.020 \\
1972.94 & \pm 0.005 \\
1982.964 & \pm 0.0005 \\
1984.130 & \pm 0.002 \\
1995.843 & \pm 0.0005 \\
2005.745 & \pm 0.005 \\
2007.692 & \pm 0.0005
\end{tabular}

